The Astrophysical Journal, 677:1253-1267, 2008 April 20

(C) 2008. The American Astronomical Society. All rights reserved. Printed in U.S.A.

\title{
MILLIARCSECOND $N$-BAND OBSERVATIONS OF THE NOVA RS OPHIUCHI: FIRST SCIENCE WITH THE KECK INTERFEROMETER NULLER
}

\author{
R. K. Barry, ${ }^{1,2}$ W. C. Danchi, ${ }^{1}$ W. A. Traub ${ }^{3}$ J. L. Sokoloski,${ }^{4}$ J. P. Wisniewski, ${ }^{1}$ E. Serabyn, ${ }^{3}$ M. J. Kuchner, ${ }^{1}$ \\ R. Akeson, ${ }^{5}$ E. Appleby, ${ }^{6}$ J. Bell, ${ }^{6}$ A. Booth,${ }^{3}$ H. Brandenburg, ${ }^{5}$ M. Colavita, ${ }^{3}$ S. Crawford, ${ }^{3}$ \\ M. Creech-Eakman, ${ }^{3}$ W. Dahl,${ }^{6}$ C. Felizardo, ${ }^{5}$ J. Garcia, ${ }^{3}$ J. Gathright, ${ }^{6}$ M. A. Greenhouse, ${ }^{1}$ \\ J. Herstein, ${ }^{5}$ E. Hovland, ${ }^{3}$ M. Hrynevych, ${ }^{6}$ C. Koresko, ${ }^{3}$ R. Ligon, ${ }^{3}$ B. Mennesson, ${ }^{3}$ \\ R. Millan-Gabet, ${ }^{5}$ D. Morrison, ${ }^{6}$ D. Palmer, ${ }^{3}$ T. Panteleeva, ${ }^{6}$ S. Ragland, ${ }^{6}$ M. Shao, ${ }^{3}$ \\ R. Smythe, ${ }^{3}$ K. Summers, ${ }^{6}$ M. Swain, ${ }^{3}$ K. Tsubota, ${ }^{6}$ C. Tyau, ${ }^{6}$ E. Wetherell, ${ }^{6}$ \\ P. Wizinowich, ${ }^{6}$ J. Woillez, ${ }^{6}$ and G. Vasisht ${ }^{3}$ \\ Received 2007 August 9; accepted 2008 January 9
}

\begin{abstract}
We report observations of the nova RS Ophiuchi (RS Oph) using the Keck Interferometer Nuller (KIN), approximately 3.8 days following the most recent outburst that occurred on 2006 February 12 . These observations represent the first scientific results from the KIN, which operates in $N$ band from 8 to $12.5 \mu \mathrm{m}$ in a nulling mode. The nulling technique is the sparse aperture equivalent of the conventional coronagraphic technique used in filled aperture telescopes. In this mode the stellar light itself is suppressed by a destructive fringe, effectively enhancing the contrast of the circumstellar material located near the star. By fitting the unique KIN data, we have obtained an angular size of the mid-infrared continuum emitting material of 6.2, 4.0, or 5.4 mas for a disk profile, Gaussian profile (FWHM), and shell profile, respectively. The data show evidence of enhanced neutral atomic hydrogen emission and atomic metals including silicon located in the inner spatial regime near the white dwarf (WD) relative to the outer regime. There are also nebular emission lines and evidence of hot silicate dust in the outer spatial region, centered at $\sim 17$ AU from the WD, that are not found in the inner regime. Our evidence suggests that these features have been excited by the nova flash in the outer spatial regime before the blast wave reached these regions. These identifications support a model in which the dust appears to be present between outbursts and is not created during the outburst event. We further discuss the present results in terms of a unifying model of the system that includes an increase in density in the plane of the orbit of the two stars created by a spiral shock wave caused by the motion of the stars through the cool wind of the red giant star.
\end{abstract}

Subject headings: novae, cataclysmic variables — stars: dwarf novae — stars: individual (HD 162214, RS Ophiuchi) — techniques: interferometric — techniques: high angular resolution

\section{INTRODUCTION}

Classical novae (CNs) are categorized as cataclysmic variable stars that have had only one observed outburst - an occurrence typified by a Johnson $V$-band brightening of between 6 and 19 mag (Warner 1995). These eruptions are well modeled as thermonuclear runaways (TNRs) of hydrogen-rich material on the surface of white dwarf (WD) primary stars that, importantly, remain intact after the event. Current theory tells us that CNs can be modeled as binary systems in which a lower mass companionthe secondary - orbits the WD primary such that the rate of mass transfer giving rise to the observed eruption is very low. Recurrent novae $(\mathrm{RNe})$ are a related class of $\mathrm{CN}$ which have been observed to have more than one eruption. Like $\mathrm{CNe}, \mathrm{RN}$ events are well represented as surface TNR on WD primary stars in a binary system, but are thought to have much higher mass transfer rates commensurate with their greater eruption frequency. There are two types of systems that produce recurrent novae - cataclysmic

\footnotetext{
1 NASA Goddard Space Flight Center, Exoplanets and Stellar Astrophysics Laboratory, Greenbelt, MD 20771.

2 Department of Physics and Astronomy, The Johns Hopkins University, Baltimore, MD 21218.

3 Jet Propulsion Laboratory, California Institute of Technology, Pasadena, CA 91109.

4 Columbia University, Department of Physics, NY 10027.

5 Michelson Science Center, Caltech 100-22 Pasadena, CA 91125.

6 W. M. Keck Observatory, California Association for Research in Astronomy, 65-1120 Mamalahoa Highway, Kamuela, HI 96743.
}

variables, in which the WD accretes from a main-sequence star that orbits the WD on a timescale of hours, and symbiotic stars, in which the WD accretes from a red giant companion that orbits that WD on a timescale of years.

$\mathrm{CNe}$ and $\mathrm{RNe}$ produce a few specific elemental isotopes by the entrainment of metal-enriched surface layers of the WD primary during unbound TNR outer-shell fusion reactions. In contrast, type Ia supernovae produce most of the elements heavier than helium in the universe through fusion reactions leading to the complete destruction of their WD primary. Some theoretical models indicate that $\mathrm{RNe}$ could be a type of progenitor system for supernovae. Importantly, these theories are predicated on two critical factors: (1) the system primary must be a compact carbonoxygen core supported solely by electron degeneracy pressure and (2) there must be some mechanism to allow the WD mass to increase secularly toward the Chandrasekhar limit.

The nova RS Oph has undergone six recorded episodic outbursts of irregular interval in 1898 (Fleming 1904), 1933 (Adams \& Joy 1933), 1958 (Wallerstein 1958), 1967 (Barbon et al. 1969), 1985 (Morrison 1985) and now 2006. There are also two possible outbursts in 1907 (Schaefer 2004) and 1945 (Oppenheimer \& Mattei 1993). All outbursts have shown very similar light curves. This system is a single-line binary, symbiotic with a red giant secondary characterized as K5.7 $\pm 0.4 \mathrm{I}-\mathrm{II}$ (Kenyon \& FernandezCastro 1987) to a K7 III (Mürset \& Schmid 1999) in quiescence and a white dwarf primary in a $455.72 \pm 0.83$ day orbit about their common center of mass as measured using single-line radial 
velocity techniques (Fekel et al. 2000). Oppenheimer \& Mattei examined all the outbursts and found that $V$-band luminosity of RS Oph decreased 0.09 mag day $^{-1}$ for the first 43 days after outburst. A 2 mag drop would then require on average 22 days, establishing RS Oph as a fast nova based on the classification system of Payne-Gaposchkin (1957).

The most recent outburst of the nova RS Oph was discovered at an estimated $V$-band magnitude of 4.5 by H. Narumi of Ehime, Japan, on 2006 February 12.829 UT (Narumi et al. 2006). This is 0.4 mag brighter than its historical average AAVSO $V$-band peak magnitude so it is reasonable to take February 12.829 (JD 2453779.329) as day zero. The speed of an outburst is characterized by its $t_{2}$ and $t_{3}$ times which are the intervals in days from the visible maximum until the system has dimmed by 2 and $3 \mathrm{mag}$, respectively. For this outburst the $t_{2}$ and $t_{3}$ times are 4.8 and 10.2 days, respectively.

The distance to the RS Oph system is of importance to the interferometry community as it effects interpretation of astrometric data (see Monnier et al. 2006). There has been a good deal of disagreement in the literature with a surprisingly broad range, from as near as $0.4 \mathrm{kpc}$ (Hachisu \& Kato 2001) to as far as $5.8 \mathrm{kpc}$ (Pottasch 1967).

Barry et al. (2008a) have recently undertaken a thorough review of the various techniques that have been used to derive a distance to RS Oph and obtain a distance of $1.4_{-0.2}^{+0.6} \mathrm{kpc}$. It is this value that we adopt for astrometric calculations in this paper.

The structure of this paper is as follows. We report highresolution $N$-band observations of RS Oph using the nulling mode of the $85 \mathrm{~m}$ baseline Keck Interferometer, beginning with a discussion of the nulling mode itself in $\S 2$. We discuss the observations in $\S 3$, and the data and analysis in $\S 4$. In $\S 5$ we introduce a new physical model of the system, which unifies many of the observations into a coherent framework. The results of this paper and those of other recent observations of RS Oph are discussed in the context of this model in $\S 6$. Finally, $\S 7$ contains a summary of our major results and conclusions.

\section{THE KECK INTERFEROMETER NULLER}

The KIN is designed to detect faint emission due, e.g., to an optically thin dust envelope, at small angular distances from a bright central star (Serabyn et al. 2000). Its operation differs from a more common fringe scanning optical interferometer in that a nulling stage precedes the fringe scanning stage (Serabyn et al. 2004, 2005, 2006; Colavita et al. 2006). The basic measurement thus remains the fringe amplitude, but both the meaning of the fringe signal in relation to the source and the processing of the fringe information differ from the normal case of a standard visibility measurement. Here we provide only a brief description of the measurement process, because this has been and will be described in depth elsewhere (Serabyn et al. 2004, 2005, 2006; Colavita et al. 2006; Serabyn et al. 2008, in preparation).

To remove both the stellar signal and the thermal background in the MIR, a two stage interferometer has been developed. To implement this approach, each Keck telescope is first split into two half-apertures, to generate a total of four collecting subapertures. The starlight is first nulled on the two long $(85 \mathrm{~m})$ parallel baselines between corresponding Keck subapertures. This generates the familiar sinusoidal fringe pattern on the sky (Fig. 1), except that the central dark fringe on the star is achromatic, and fixed on the star, to achieve deep and stable rejection of the starlight. After the nulling stage, the residual, nonnulled light making it through the first stage fringe pattern is measured by a fringe scan in a second stage combiner, the "cross-combiner," which combines the light across the Keck apertures ( $\sim 4 \mathrm{~m}$ baseline).
Thus, what is measured is the fringe amplitude of this "nulled source brightness distribution" (Serabyn et al. 2008, in preparation) This quantity is then normalized by the total signal. This is measured by moving the nullers to the constructive phase, and again scanning the cross-combiners. The basic measured quantity, the null depth, $N$, is then the ratio of the signals with the star in the destructive and constructive states. $N$ is related to the classical interferometer visibility $V=\left(I_{\max }-I_{\min }\right) /\left(I_{\max }+I_{\min }\right)$, the modulus of the complex visibility $\hat{V}$, by a simple formula:

$$
N=\frac{1-V}{1+V} \text {. }
$$

The long nulling baselines produce fringes spaced at about 23.5 mas at $10 \mu \mathrm{m}$, while the short baseline produces fringes spaced at $\sim 400$ mas, which is similar to the size of the primary beam and is assumed to be large compared to the extent of the target object. Modulating its phase therefore modulates the transmitted flux of the entire astronomical source, as modified by the sinusoidal nuller fringes, and so the amplitude of this modulated signal gives the flux that passes through the fringe transmission pattern produced by the long-baseline nullers.

\section{OBSERVATIONS}

We observed the nova about an hour angle of about -2.0 on the Keck Interferometer in nulling mode on 2006 Feb 16 with a total of three observations between day 3.831 and 3.846 postoutburst bracketed with observations of two calibrators stars, $\rho$ Boo and $\chi$ UMa. Data were obtained at $N$ band $(8-12.5 \mu \mathrm{m})$ through both ports of the KALI spectrograph with the gating of data for long-baseline phase delay and group delay turned on. The Infrared Astronomical Satellite (IRAS) Low Resolution Spectrometer (LRS) spectra for the calibrator stars were flux-scaled according to the broadband IRAS $12 \mu \mathrm{m}$ fluxes. The nova data were flux calibrated and telluric features were removed using calibrator data. These calibrators are well matched to the target flux, and their size and point symmetry are well known. A journal of observations is presented in Table 1.

Our data analysis involves removing biases and coherently demodulating the short-baseline fringe with the long-baseline fringe tuned to alternate between constructive and destructive phases, combining the results of many measurements to improve the sensitivity, and estimating the part of the null leakage signal that is associated with the finite angular size of the central star. Comparison of the results of null measurements on science target and calibrator stars permits the instrumental leakage - the "system null leakage"- to be removed and the off-axis light to be measured.

Sources of noise in the measurements made by this instrument have been well described elsewhere (Koresko et al. 2006); however, we outline them here for reference. The null leakage and intensity spectra include contributions from the astrophysical size of the object, phase and amplitude imbalances, wave front error, beam train vibration, pupil polarization rotation, and pupil overlap mismatch. There are also biases, mostly eliminated by use of sky frames, in the calculated fringe quadratures, caused mainly by thermal background modulation due to residual movement of the mirrors used to shutter the combiner inputs for the long baselines. Another source of error is the KALI spectrometer channel bandpass, which is large enough to produce a significant mismatch at some wavelengths between the center wavelength and the shortbaseline stroke OPD. This effect, termed warping, distorts the quadratures and is corrected by a mathematical dewarping step accomplished during calibration. There is also the effect of the 


\section{Keck Interferometer Nuller}
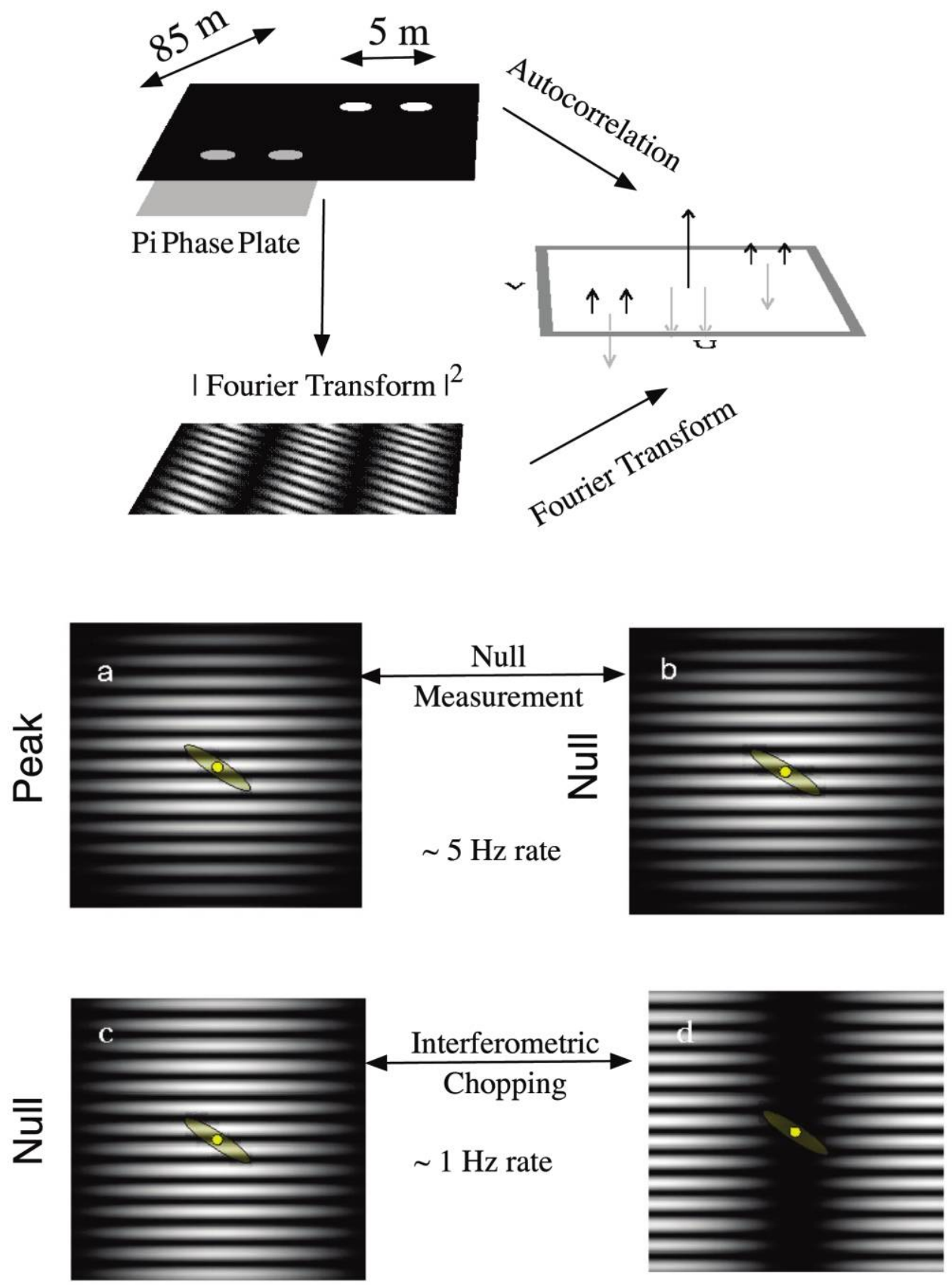

Fig. 1.- Top part of plot displays a conceptual view of the operation of the KIN system. There are two short baselines, separated by $5 \mathrm{~m}$, and two long baselines separated by $85 \mathrm{~m}$. A $\pi$ phase shift is applied to the pupils on the long baseline. The autocorrelation of the four pupils is shown, as well as the equivalent intensity pattern, also called the transmission pattern. Radiation passing through the white striped regions is detected by the system. The bottom part of the plot displays the sequence used for the measurement process. There are two chopping sequences: $(a)$ and $(b)$ display the $\sim 5 \mathrm{~Hz}$ chopping between the null and bright fringe patterns on the source using the $85 \mathrm{~m}$ baseline for starlight subtraction, used to measure the null response, while $(c)$ and $(d)$ show the $\sim 1 \mathrm{~Hz}$ chop sequence between the two short baselines used to remove the telescope and sky backgrounds. Note that the long-baseline fringes are not to scale with the field of view in this depiction.

partial resolution of extended structure on the long baselines, which will cause the flux to be undercounted by some amount depending on the spatial extent and distribution of the emitting region. Compared to the error bars this is a rather small effect for most normal stars, and is unlikely to have much influence on the actual spectrum, however, unless the emission lines are coming from a very extended shell approaching 25 mas in angular size. We do not expect this to be the case for nova RS Oph at day 3.8.

The most important contributor to measurement noise is sky and instrument drifts between target and calibrator. Other potential 
TABLE 1

OBSERVING LOG FOR RS OPHIUCHI

\begin{tabular}{cccccc}
\hline \hline Object & Type & $\begin{array}{c}\text { Time } \\
(\mathrm{UT})\end{array}$ & $\begin{array}{c}U \\
(\mathrm{~m})\end{array}$ & $\begin{array}{c}V \\
(\mathrm{~m})\end{array}$ & Air Mass \\
\hline$\chi$ UMa ................ & Calibrator & $15: 07: 07$ & 23.85 & 80.61 & 1.38 \\
$\chi$ UMa ................ & Calibrator & $15: 15: 39$ & 21.92 & 81.25 & 1.41 \\
RS Oph ............... & Target & $15: 50: 15$ & 54.57 & 64.75 & 1.46 \\
RS Oph ............... & Target & $16: 03: 46$ & 55.35 & 64.37 & 1.39 \\
RS Oph ............... & Target & $16: 12: 35$ & 55.75 & 64.12 & 1.35 \\
$\rho$ Boo .................. & Calibrator & $16: 34: 24$ & 39.63 & 75.14 & 1.08 \\
\hline
\end{tabular}

sources of noise include the difference between the band center wavelength for the interferometer and that of the IRAS LRS calibrator, undercounting of stellar flux resulting from glitches in the short-baseline phase tracking, and the chromaticity of the first maximum of the long-baseline fringe. None of these are significant for the following reasons. First, the calibration is based not on broadband photometry but on the IRAS LRS spectrum. Second, short-baseline tracking glitches happen nearly as frequently on target observations as on calibrator observations, so they should have minimal effect on overall calibrated flux. Third, the effect of chromaticity should be negligible because fringe detection is done on a per-spectral-channel basis. As a result, it is only affected by dispersion within the individual KALI spectral channels, which are about $0.3 \mu \mathrm{m}$ in width.

\section{DATA AND ANALYSIS}

We developed a mathematical solution and software suite to model the observatory and source brightness distribution. We used this suite to conduct an exhaustive grid search and to generate a Monte Carlo confidence interval analysis of solution spaces of these models. We explored three types of models for the source surface brightness distribution; Gaussian, disk, and shell. Limited $(u, v)$ coverage permitted only rotationally symmetric models with two parameters - size and flux. We used $\chi^{2}$ minimization to obtain the best-fit models for both the inner and outer spatial regimes simultaneously. Table 2 displays size measurements, flux values, and $1 \sigma$ confidence interval values. The error bars have been increased slightly beyond the $\chi^{2}$ values to incorporate the effect of an adopted $0.0051 \sigma$ systematic error which would be correlated among measurements at different wavelengths.

The measurements made with the two KALI ports are somewhat independent - the data they produce are combined for purposes of fringe tracking, but not for data reduction. The system null and the final calibrated leakage are computed separately for the two ports. The apparent inconsistency detected between the ports is the result of optical alignment drifts at the time of the measurement. In particular, the last calibrator measurement showed a sudden change in the system null for port 1 , while for all the other calibrator measurements the system nulls were stable. We therefore compared our source brightness distribution models against KALI port 2 data alone. For the best-fit models in Table 2 we used Spitzer spectra to identify and remove emission features centered at 8.7, 9.4, 10.4, 11.4, and $12.5 \mu \mathrm{m}$ in the KIN inner and $8.9,9.8$, and $11.4 \mu \mathrm{m}$ in the KIN outer spatial regime. We removed the emission feature data because our intention was to model the continuum.

Figure 2 shows two sets of $8-12.5 \mu \mathrm{m}$ spectra of RS Oph on day 3.8 postoutburst. The upper plot shows the outer spatial regime, which is the dimensionless null leakage spectrum, i.e., the intensity of light remaining after destructive interference divided by the intensity spectrum, plotted against wavelength in microns. The lower plot is the intensity spectrum, which is light principally from the inner 25 mas centered on the source brightness distribution orthogonal to the Keck Interferometer baseline direction $-38^{\circ}$ east of north. The null leakage spectrum may be broadly described as a distribution that drops monotonically with increasing wavelength overlaid with wide, emission-like features. The intensity spectrum, with an average flux density of about 22 Jy over the instrument spectral range, may be similarly described but with a continuum that has a saddle shape with a distinct rise at each end. The underlying shape curves upward for wavelengths shorter than approximately $9.7 \mu \mathrm{m}$ and longer than $12.1 \mu \mathrm{m}$. Overlaid on each of these are traces representing simple models of source brightness distributions fit to the data, described above.

Figure 3 shows the inner and outer spatial regimes from the KIN data together with Spitzer data from day 63 (Evans et al. 2007a). The absolute fluxes of these data are unscaled and given with a broken ordinate axis for clarity. The outer KIN spectrum flux has been multiplied by 2 to correct for the transmission through the fringe pattern for extended sources. In efforts to identify the sources of these emission features we took the high-resolution Spitzer spectra and, using boxcar averaging, rebinned the data until it and the KIN data had equivalent resolution. The binned Spitzer spectrum is quite similar in character to the KIN spectra. Importantly, the sum of the inner and outer spatial regime KIN spectra is nearly identical to the binned Spitzer spectrum with the exception that the absolute scale magnitude is, on day 3.8, about an order of magnitude greater than that of the Spitzer spectrum. This is as expected because the measured flux drops with time after the peak due to cooling and the summed inner and outer spatial regime data should very nearly reproduce the transmission of a filled aperture telescope of equivalent diameter.

The wavelength range sampled by the KALI spectrometer, $8-12.5 \mu \mathrm{m}$, covers many important discrete transitions including molecular rotation-vibration, atomic fine structure, and electronic transitions of atoms, molecules, and ions. This range also samples several important transitions in solids such as silicates found in dust and polycyclic aromatic hydrocarbons (PAHs). With the

TABLE 2

RS Ophiuchi Model Fitting Results

\begin{tabular}{|c|c|c|c|c|}
\hline \multirow[b]{2}{*}{ Source Model } & \multirow{2}{*}{$\begin{array}{c}\text { Angular Size ( } N \text { Band) } \\
\text { (mas) }\end{array}$} & \multirow{2}{*}{$\begin{array}{c}\text { Radiant FluX } \\
\text { (Jy) }\end{array}$} & \multicolumn{2}{|c|}{$K$ BAND (mas) } \\
\hline & & & Major Size & Minor Size $\mathrm{a}^{\mathrm{a}}$ \\
\hline Uniforr & $6.2 \pm 0.6$ & $22.4 \pm 3.9$ & $4.9 \pm 0.4$ & $3.0 \pm 0.3$ \\
\hline Uniform Gaussian ${ }^{\mathrm{b}}$. & $4.0 \pm 0.4$ & $22.4 \pm 3.8$ & $3.1 \pm 0.2$ & $1.9 \pm 0.3$ \\
\hline Uniform shell ${ }^{\mathrm{c}} \ldots \ldots \ldots \ldots \ldots \ldots \ldots \ldots$ & $5.4 \pm 0.6$ & $22.4 \pm 3.8$ & $3.7 \pm 0.3$ & $1.9 \pm 0.2$ \\
\hline
\end{tabular}

${ }^{\text {a }}$ Sizes for continuum values at $2.3 \mu \mathrm{m}$ after Chesneau et al. (2007).

${ }^{\mathrm{b}}$ FWHM.

${ }^{c}$ Spherical shell with thickness 1.0 mas-optically thin. 

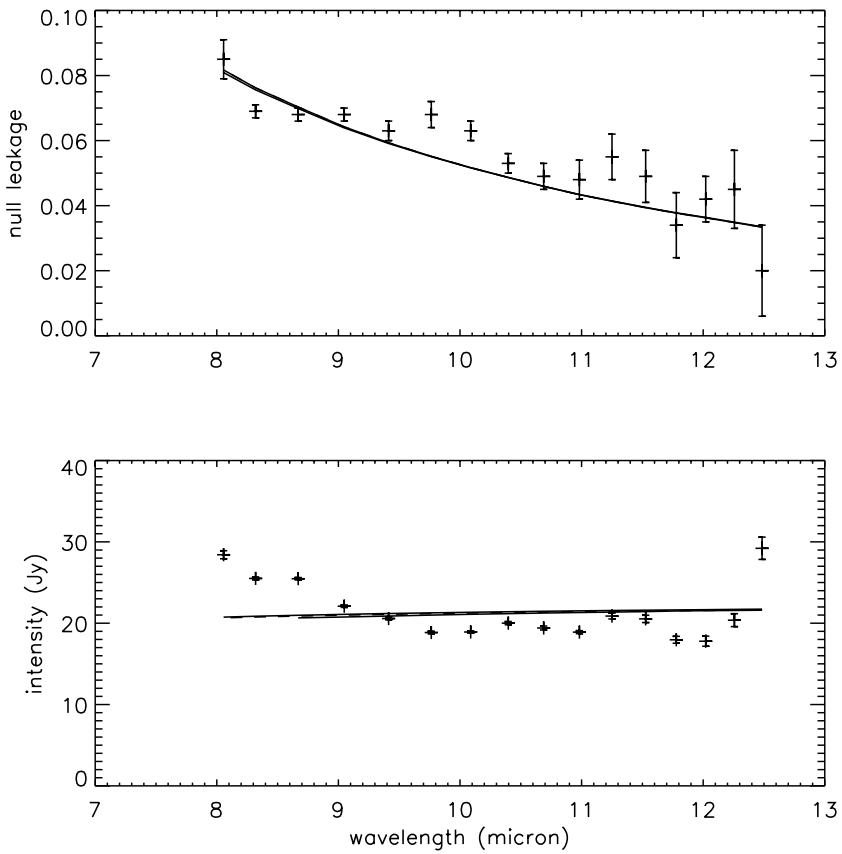

FIG. 2.-Plot of three best-fit continuum models against KIN data. All three models; disk, shell, and Gaussian, that minimized $\chi^{2}$ simultaneously against the null leakage and intensity spectra, lie effectively on top of one another. The top trace gives the dimensionless null leakage or interferometric observable, which is the null fringe output divided by the intensity spectrum. The lower trace is the constructive fringe output or intensity spectrum. As described in the text we have removed several data points associated with emission features from the original set for the purpose of fitting the continuum.

exception noted below, spectral features in the KIN data are clearly not resolved by the instrument and are often, in the associated Spitzer spectrum, Doppler broadened and blended. Also, because the Spitzer spectra were not taken contemporaneously with the KIN spectra and because of the transient nature of the $\mathrm{RNe}$, identification of KIN features with Spitzer lines is necessarily tentative.

Figure 4 shows Spitzer spectra on days 63, 73, and 209 after peak $V$-band brightness. The continuum decreases monotonically with time. The continuum values are approximately $1.4,1.1$, and $0.25 \mathrm{Jy}$ for April 14, April 26, and September 9, respectively. The first two spectra on April 14 and 16 show strong atomic lines with no obvious evidence of dust emission. However, it is expected that thermal bremsstrahlung from the central source will overwhelm any other faint sources. In addition, there are several narrow emission features in this latter spectrum which appear to be similar to those in the earlier spectra. In contrast, the spectrum taken on September 9 shows a distinct broad emissions feature from 8.9 to $14.3 \mu \mathrm{m}$ peaking at $10.1 \mu \mathrm{m}$. We proceed on the assumption that this is emission from dust in the vicinity of the nova.

Referring again to the KIN spectrum in Figure 3, strong continuum radiation is apparent in both inner and outer spatial regimes. In contrast, while the continuum was still clearly visible in $J, H, I, K$ bands on February 24 and detectable on April 9 (Evans et al. 2007c), it has subsided by day 63 in Figures 3 and 4 . Comparing the spectra from RS Oph to those from V1187 Sco (Lynch et al. 2006) note that the continuum given for V1187 Sco is non-Planckian showing an excess longward of $9 \mu \mathrm{m}$ and is strongly red as compared to an F5 V Kurucz spectrum. The V1187 Sco and RS Oph continua have slopes that agree to within $10 \%$. While both free-bound and free-free transition processes lead to emission of continuum radiation, in the MIR spectral range thermal bremsstrahlung free-free emission dominates. We attribute

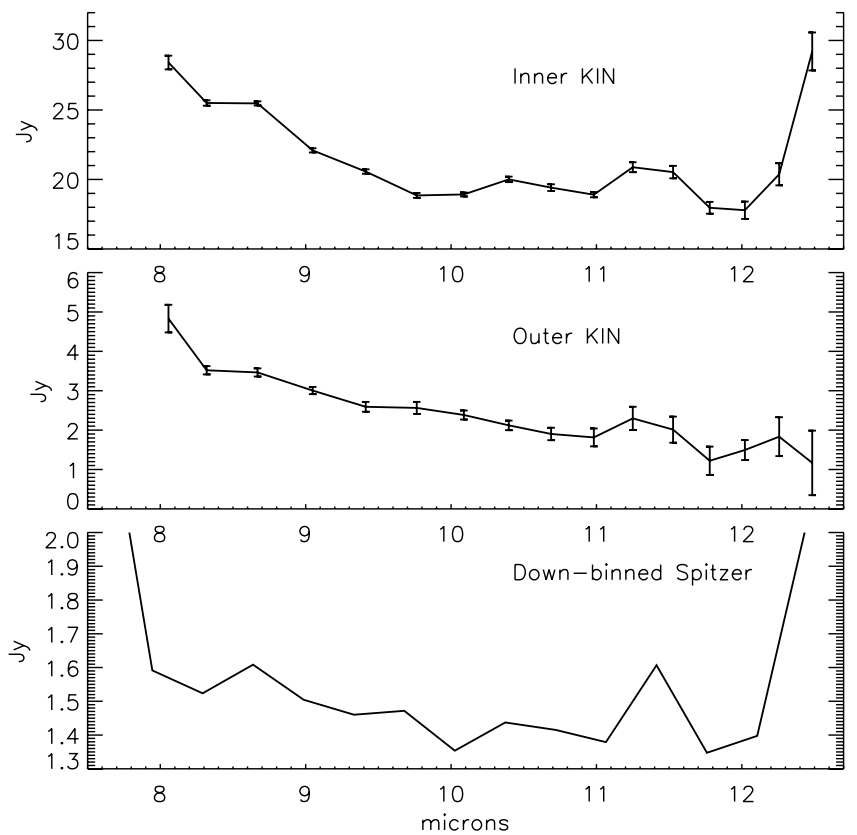

FIG. 3.-Plot of KIN spectra together with a Spitzer spectrum. Upper trace is the intensity spectrum, which is the inner KIN spatial regime-light dominated by the inner 25 mas about the center of the source brightness distribution at midband. The middle trace is the nulled fringe output which is the interferometric observable times the intensity spectrum multiplied by 2 (to correct for transmission through the fringe pattern for extended sources) to give the source brightness in the outer spatial regime. This is predominantly emission from material greater than about 12.5 mas $(\sim 17 \mathrm{AU}$ at $1.4 \mathrm{kpc})$ from the center of the source. The lower trace is Spitzer data (Evans et al. 2007b) from day 63 boxcar averaged to yield approximately the same spectral resolution as the KIN. None of the data were continuum normalized. Note the features between 9 and $11 \mu \mathrm{m}$ and how the inner and outer spatial regime spectra are different from one another. Note that the Spitzer spectra have very low spatial resolution and combine the light from the entire region detected by both KIN inner and outer spatial regimes.

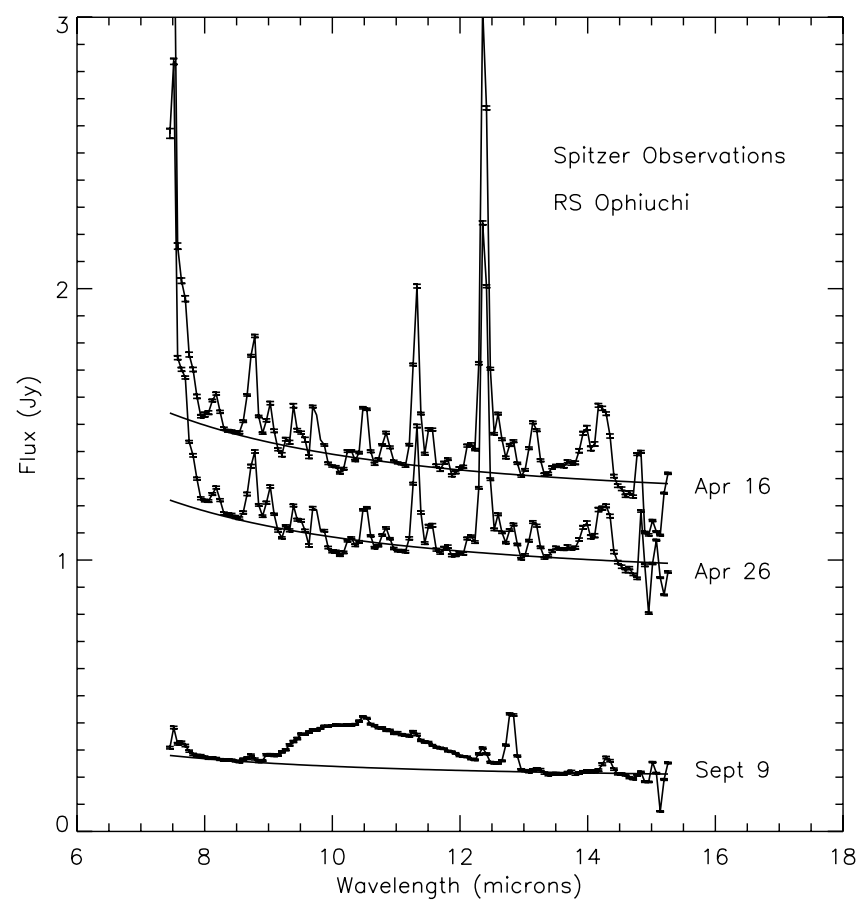

FIG. 4.-Spitzer space telescope spectra from days 63, 73, and 209. Here we see that the continuum drops rapidly as time advances with the spectral emission features almost identical on April 16 and April 26. Data obtained on September 9 is starkly different with a strong solid state feature evident between 9 and $13 \mu \mathrm{m}$ (see Evans et al. [2007c] for more details on the Spitzer data). 
TABLE 3

Mid-Infrared Spitzer Line List: $N$-Band

\begin{tabular}{|c|c|c|c|c|}
\hline $\begin{array}{l}\text { Wavelength } \\
\qquad(\mu \mathrm{m})\end{array}$ & ID & $\begin{array}{c}\text { April } 16 \\
\text { (Jy) }\end{array}$ & $\begin{array}{c}\text { April } 26 \\
\text { (Jy) }\end{array}$ & $\begin{array}{c}\text { September } 9 \\
(\mathrm{Jy})\end{array}$ \\
\hline $7.460 \ldots \ldots \ldots \ldots$ & Pf $\alpha-\mathrm{H}_{\text {г }}: 6-5$ & Detected $^{\mathrm{a}}$ & Detected & Detected \\
\hline $7.652 \ldots \ldots \ldots \ldots$ & {$[\mathrm{Ne} \mathrm{VI}]$} & Detected & Detected & Detected \\
\hline $8.180 \ldots \ldots \ldots \ldots . . . . .$. & $\mathrm{Fe}_{\mathrm{I}}$ & 0.009 & 0.007 & - \\
\hline $8.760 \ldots \ldots \ldots \ldots . . . .$. & Н г : $10-7$ & 0.042 & 0.038 & $0.020^{\mathrm{b}}$ \\
\hline $8.985 \ldots \ldots \ldots \ldots$ & {$\left[\mathrm{V}\right.$ II], $\mathrm{Si}_{\mathrm{I}}$, and $\left.\mathrm{Ca}_{\mathrm{I}}\right]$} & $\ldots$ & $\ldots$ & 0.010 \\
\hline $9.017 \ldots \ldots \ldots \ldots$ & $\mathrm{Fe}_{\mathrm{I}}$ & 0.015 & 0.021 & $\ldots$ \\
\hline $9.288 \ldots \ldots \ldots \ldots$ & $\mathrm{Ca}_{\mathrm{I}}$ & 0.004 & 0.004 & $\ldots$ \\
\hline $9.407 \ldots \ldots \ldots \ldots . . .$. & $\mathrm{Si}$ I & 0.014 & 0.013 & $\ldots$ \\
\hline $9.529 \ldots \ldots \ldots \ldots$ & $\mathrm{C}_{\mathrm{I}}$ & 0.009 & 0.008 & $\ldots$ \\
\hline $9.720 \ldots \ldots \ldots \ldots . . .$. & $\mathrm{C}_{\text {II }}$ & 0.018 & 0.017 & $\ldots$ \\
\hline $9.852 \ldots \ldots \ldots \ldots . .$. & $\mathrm{Ca}$ I & 0.007 & 0.008 & $\ldots$ \\
\hline $10.285 \ldots \ldots \ldots$. & $\mathrm{Mg}_{\mathrm{I}}$ & 0.007 & 0.006 & $\ldots$ \\
\hline $10.492 \ldots \ldots \ldots . .$. & $\mathrm{H}_{\mathrm{I}}: 12-8$ & $\ldots$ & $\ldots$ & 0.013 \\
\hline $10.517 \ldots \ldots \ldots$ & $\mathrm{Ne}_{\mathrm{I}}$ & 0.026 & 0.026 & $\ldots$ \\
\hline $10.833 \ldots \ldots \ldots . . .$. & $\left.\mathrm{C}_{\mathrm{I}}\right]$ & 0.018 & 0.016 & $\ldots$ \\
\hline $11.284 \ldots \ldots \ldots . . . . .$. & Н г : $9-7$ & $\ldots$ & $\ldots$ & 0.007 \\
\hline $11.318 \ldots \ldots \ldots . .$. & $\mathrm{He}_{\mathrm{I}}$ & 0.060 & 0.053 & $\ldots$ \\
\hline $11.535 \ldots \ldots \ldots . .$. & He II & 0.016 & 0.013 & $\ldots$ \\
\hline $12.168 \ldots \ldots \ldots . . .$. & $\mathrm{H}_{\mathrm{I}}: 36-11$ & 0.011 & 0.009 & $\ldots$ \\
\hline $12.372 \ldots \ldots \ldots$ & Нu $\alpha-\mathrm{H}_{\text {I }}: 7-6$ & 0.158 & 0.150 & 0.037 \\
\hline $12.557 \ldots \ldots \ldots$ & $\mathrm{Si}_{\mathrm{I}}$ & 0.040 & 0.036 & $\ldots$ \\
\hline $12.803 \ldots \ldots \ldots . .$. & {$[\mathrm{Ne} \mathrm{II}]$} & $\ldots$ & $\ldots$ & 0.159 \\
\hline $12.824 \ldots \ldots \ldots . .$. & $\mathrm{He}_{\mathrm{I}}$ & 0.009 & 0.015 & $\ldots$ \\
\hline $13.128 \ldots \ldots \ldots \ldots$ & $\mathrm{He}$ II & 0.009 & 0.011 & $\ldots$ \\
\hline $13.188 \ldots \ldots \ldots . . . .$. & Н I : $18-10$ & 0.009 & 0.010 & 0.005 \\
\hline
\end{tabular}

Notes.-Some of these species were blended with others, and, due to the difficulty in deblending Spitzer low-resolution channel spectra, may in some cases be misidentified. This is not critical, however, to the conclusions of this paper.

${ }^{a}$ Detected in Spitzer spectrum but not fit due to intrusion of band edge.

${ }^{\mathrm{b}}$ Blended with neutral hydrogen lines at 8.721 and $8.665 \mu \mathrm{m}$. the drop in continuum radiation to the transition to line-emission cooling mechanisms. Also, by the time Spitzer data were taken, the object had become less dense and so the emission coefficient for thermal bremsstrahlung (proportional to number density of protons and electrons) had dropped considerably. The continuum emission is described in detail by Barry et al. (2008b).

Table 3 gives identification of all narrow features in Figure 4 where it is possible to do so, as follows. We generated line lists of atomic species assuming that all ionization stages of all elements were possible for the three Spitzer spectra. We assumed standard cosmic abundances (Grevesse 1984). After continuum normalization, each emission line was fitted with a Gaussian and, where necessary, de-blended using IRAF. Our fitted emission features were compared with the Spitzer list.

Table 4 gives our identification of particular emission sources with the continuum-normalized spectral features in the KIN inner and outer spectra. The center wavelength of each of the broad features is listed in the first column, while the second column displays a width for each feature defined as the cutoff wavelength at the intersection of the feature and the unity continuum linethe full-width zero intensity (FWZI) level of the feature. The flux and the $1 \sigma$ uncertainty contained in that feature through measurement of the total area under it and above the unity continuum level is in the third column. The fourth column shows the particular atomic species found in the Spitzer spectrum as identified with each KIN spectral feature. Because Spitzer lacks the spatial resolution to discriminate the inner from the outer regions of the nova and the KIN lacks the spectral resolution to discriminate among atomic species we assumed that features in the KIN spectra would most reasonably be identified with those in Spitzer spectra from 2006 April and September, respectively. In particular, Spitzer emission lines identified with corresponding ones in KIN inner spatial regime, dominated by light originating in the close vicinity the WD, are well represented by a cosmic distribution of atomic elements. We assumed that any condensates within the blast radius of

TABLE 4

Continuum-normalized, Mid-infrared Kin Emission Source Identification

$\begin{aligned} & \text { Center Wavelength } \\ & (\mu \mathrm{m})\end{aligned}$
$\begin{aligned} & \text { Spectroscopic Width } \\ & (\mu \mathrm{m}, \mathrm{FWZM})\end{aligned}$


TABLE 5

Post-outburst Evolution of the White Dwarf Star in RS Oph

\begin{tabular}{|c|c|c|}
\hline $\begin{array}{r}\text { Time }^{\mathrm{a}} \\
\text { (days) }\end{array}$ & $\begin{array}{l}\text { Radius } \\
\left(R_{\odot}\right)\end{array}$ & $\begin{array}{c}\text { Temperature } \\
\text { (K) }\end{array}$ \\
\hline$\ldots \ldots \ldots \ldots \ldots$ & 45 & 12,200 \\
\hline 2 & 21 & 20,000 \\
\hline 14 & 1.6 & 67,000 \\
\hline - & 0.56 & 11,4000 \\
\hline 51 & 0.23 & 18,1000 \\
\hline 72 & 0.083 & 302,000 \\
\hline 119 & 0.0037 & $1,020,000$ \\
\hline 251 & 0.003 & 350,000 \\
\hline
\end{tabular}

Nоте.-Stellar parameters for the white dwarf star after the 2006 outburst of RS Oph based on Hachisu \& Kato 2001.

${ }^{a}$ The zero time for these parameters is the $V$-band maximum light, which occurs about 3-4 days after the thermonuclear runaway process begins.

the nova would be sublimated away and dissociated into atoms, and that any nucleosynthesis that occurred in the outer layers of the WD during TNR would negligibly impact abundances. The KIN inner spatial regime spectral features were keyed to Spitzer spectral features in the April 16 and 26 spectra. Similarly, the KIN outer spatial regime, light predominantly from a region $\sim 17 \mathrm{AU}$ from the WD, is keyed to the Spitzer spectrum taken on September 9 (Evans et al. 2007b) as it is assumed that the abundances of atomic species in the latter, nebular Spitzer spectrum, would reasonably be representative of the environment observed by that KIN channel.

Note that other symbiotic novae (e.g., V1016 Cyg, RR Tel) have broad emission features around 10 and $18 \mu \mathrm{m}$, evident in our Spitzer spectrum, that have been successfully fitted by crystalline silicate features (e.g., Sacuto et al. 2007; Schild et al. 2001; Eyres et al. 1998). Motivated by this fact, we calculated the temperature and emission SED of various species of dust at the range from the pseudophotosphere of the WD at which the KIN's outer spatial regime has maximum transmission. The center of the first constructive fringe, when the null fringe is located on top of the WD, at $9.8 \mu \mathrm{m}$ and a projected baseline of $84 \mathrm{~m}$, is $\sim 12$ mas from the WD. At a distance to the object of $1.4 \mathrm{kpc}$ (Barry et al. 2008a) this corresponds to about $17 \mathrm{AU}$. At day 3.8, the pseudophotosphere of the WD has a luminosity of $1.6 \times 10^{5} L_{\odot}$ (radius, $18.1 R_{\odot}$, and temperature, $27,050 \mathrm{~K}$ ), computed by interpolating between values for the postoutburst evolution displayed in Table 5. The temperature of a black dust grain in thermal equilibrium is $272 \mathrm{~K}$, using equation (A3) from the Appendices in this paper, and is well below the sublimation temperature of silicate dust, $\sim 1500 \mathrm{~K}$. The dust feature in the Spitzer September 9 spectrum is wide enough at FWHM that it falls across over seven spectroscopic elements in the KIN outer spatial channel meaning that the dust is both spectrally resolved and spatially localized.

Figure 5 displays the association of identified Spitzer atomic emission lines with the continuum-normalized KIN inner and outer regime spectral features. Clearly the two traces are markedly different. Note that when the inner and outer spatial regimes are summed, the result closely follows the rebinned 2006 April 16 Spitzer spectra with the exception of the KIN outer spatial regime feature (lower trace) centered at $9.8 \mu \mathrm{m}$. Based on our assumptions of a primarily nebular environment in the vicinity of the outer spectrum, the atomic metals evident in the upper trace (KIN Inner spatial regime) would be unlikely to contribute to this feature. In any case, the total power in these metal lines in the wavelength range of this feature (see Table 3), including Ca I, Si I, C I, and $\mathrm{C}$ II, evident in the upper trace is $0.05 \mathrm{Jy}$, while that in the
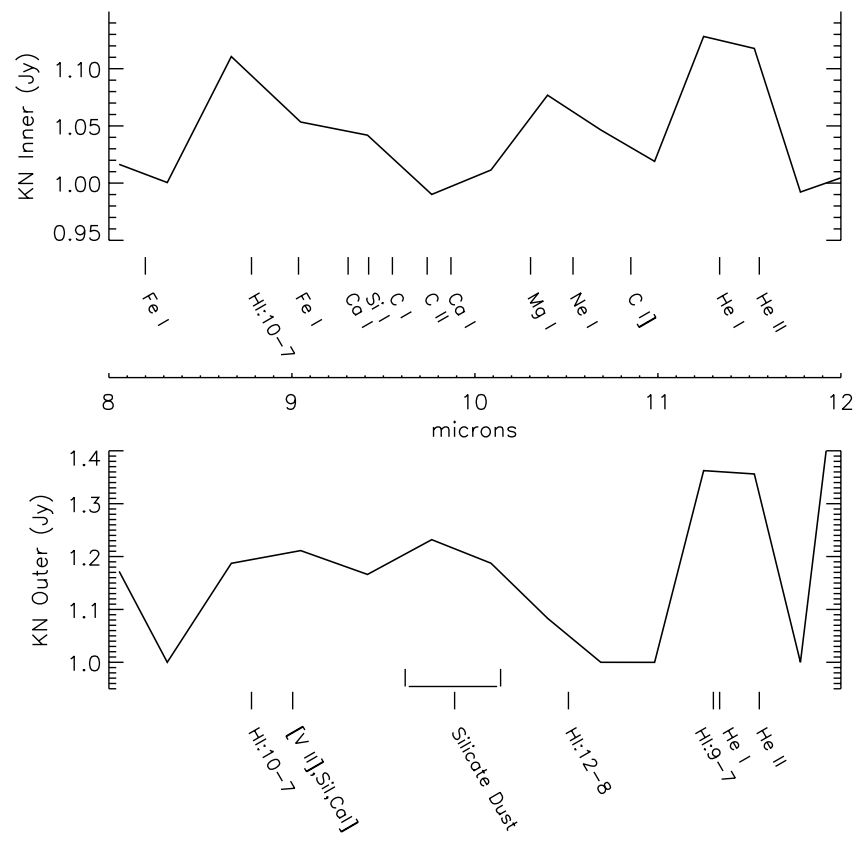

FIG. 5.-Continuum normalized KIN spectra 3.8 days after peak $V$-band brightness. Spectra are shown matched with lines identified in Spitzer spectra. The inner spatial regime line identification was matched to the cosmic abundances seen in the earlier Spitzer spectra while the outer spatial regime was biased toward the later spectrum on 2006 September 9 that included a solid state feature and were assumed to have primarily nebular abundances. Please see the text for more discussion of the line identification process.

broad feature centered at $9.8 \mu \mathrm{m}$ in the lower trace exhibits $0.24 \mathrm{Jy}$. Our models suggest that the source of this feature may be hot silicate dust in the temperature range $800-1000 \mathrm{~K}$.

Note that our KIN data detects the faint emission from well outside of the blast radius, assuming an initial shock front velocity of $3500 \mathrm{~km} \mathrm{~s}^{-1}$ (O’Brien et al. 2006) and negligible deceleration. The radiation from this spatial region originates primarily from material around the nova that has been illuminated and warmed by photons from the nova flash and, as a result, must have existed before the nova event. This establishes that silicate dust, created in the vicinity of the RS Ophiuchi system some time previous to the 2006 outburst, is detected by our measurements, and is consistent with the conclusions of Evans et al. (2007b).

\section{A PHYSICAL MODEL OF THE RECURRENT NOVA}

One aspect of the RS Oph binary system that has been neglected in the current literature regarding the recent outburst is the effect of the motion of the two stars through the wind created by mass loss from the red giant star. Garcia (1986) suggested that there could be a possible ring of material of diameter less than $40 \mathrm{AU}$ around the RS Oph binary system or possibly surrounding the red giant component, based on his measurements of an absorption feature in the core of the $\mathrm{Fe}$ II emission line profile at $5197 \AA$. The observations were performed in 1982 and 1983, several years before the 1985 nova outburst.

Subsequently, and motivated by somewhat different observations, Mastrodemos \& Morris (1999) computed three-dimensional hydrodynamical models of morphologies of the envelopes of binaries with detached WD and RG/AGB components in general. Their purpose was to see if these models could reproduce some of the observed characteristics of axisymmetric or bipolar preplanetary nebulae. Their study focused on a parameter space that encompassed outflow velocities from 10 to $26 \mathrm{~km} \mathrm{~s}^{-1}$, circular orbits with binary separations from 3.6 to $50 \mathrm{AU}$, and binary 


\section{RS Ophiuchi Before Nova}

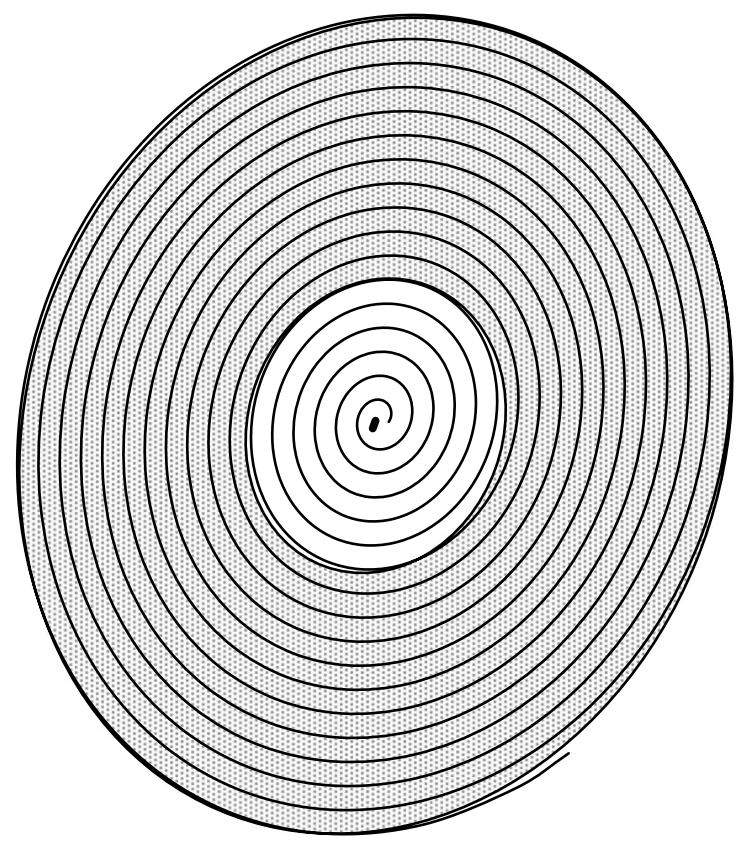

100 mas

FIG. 6.-Proposed model of circumstellar material surrounding the binary RS Oph before the nova eruption. The interaction of the white dwarf and red giant star in the slow dense wind of the red giant star can create a spiral shock with an enhanced density in the plane of the orbit of the two stars. The overall size of the dense in-plane material is of the order of 100 mas if RS Oph is at a distance of $1400 \mathrm{pc}$ and the wind speed is about $20 \mathrm{~km} \mathrm{~s}^{-1}$.

companions having a mass range of $0.25-2 M_{\odot}$. For binary separations of about 3.6 AU and mass ratios of 1.5, it was possible to generate a single spiral shock that winds $2-3$ times around the binary before it dissipates at $>25$ times the radius of the RG star. There is a density enhancement of about a factor of 100 over the normal density in the wind in the plane of the orbit of the two stars, and an under density or evacuated region perpendicular to the plane of the orbit. Observational support for this model was found recently by Bode et al. (2007), who detected a double-ring structure in HST data, which they interpreted as due to an equatorial density enhancement and by Mauron \& Huggins (2006), who observed a spiral pattern around the AGB star AFGL 3068 both of which were consistent with the model of Mastrodemos \& Morris. The underlying binary, a red giant and white dwarf, was discovered by Morris et al. (2006), who also determined the binary separation and hence approximate orbital period, which was consistent with expectations from the appearance of the spiral nebula pattern seen by Mauron \& Huggins and the model.

Figure 6 shows the proposed geometry of the nebula in the plane of the orbit of the RS Oph system based on the parameters adopted from Dobrzycka \& Kenyon (1994) for the system, including an orbital period of 460 days, and an inclination angle of about $33^{\circ}$, for the epoch just prior to the nova outburst. The spiral shock model produces an Archimedian spiral nebula, with the separation

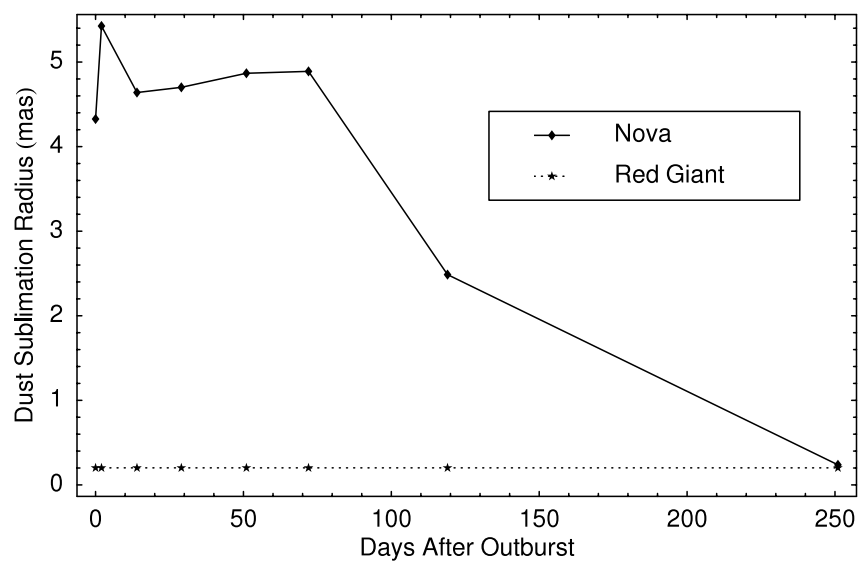

FIG. 7.- Sublimation radius of dust (mas) as a function of the number of days postoutburst for the nova and for the red giant companion.

between adjacent windings of about 3.3 mas based on the period noted above and a wind speed from the red giant star of $20 \mathrm{~km} \mathrm{~s}^{-1}$. Based on these parameters we estimate that about 17 such rings could be created between outbursts, with the overall size of the nebula of the order of 100 mas. However, there could be as few as 10 rings to as many as about 20 rings, depending on the parameters, some of which are not well known.

In order to better understand the Keck Nuller observations and other high angular resolution observations, we have modeled the evolution of the circumstellar nebula following the outburst, which we display in Figure 9. For the purposes of this discussion, we adopt the model of Hachisu \& Kato (2001), but with the simplification of a flat accretion disk geometry, i.e., not warped as in their paper. The details of our calculations are presented in the Appendix to this paper.

The results are of fundamental importance to the interpretation of our KIN data. We begin with the most immediate effect after the blast, which is the sublimation of dust within a zone where the temperature of a blackbody dust grain would be $>1500 \mathrm{~K}$ in equilibrium. Figure 7 displays the evolution of the dust sublimation radius, which is roughly $4-5$ mas until about day 70 , after which it steadily declines to $\ll 1$ mas about 250 days after the outburst. This means that much or all of the dust within this zone has entered the gaseous phase, except for a small "sliver" of material in the shadow of the red giant star. This provides additional hot gas (rich with metals) that is subsequently affected by the blast wave passing through within the next few days.

As the two stars move relative to each other in their orbit about their common center of mass, the location of the shadow of the nova moves, and consequently the material in the shadow that has not been affected by the blast wave from the nova will be sublimated during this luminous phase, creating hot gas in the vicinity within a few mas of the stars. This material may still have the type of repeating density structure that was initially present, and may be observable with high angular resolution instrumentation.

Whether or not this particular material is observable depends in part on the density distribution as a function of latitude of the plane of the orbit of the two stars. If the material is uniformly distributed over $4 \pi$ steradians, then the fraction of the total solid angle subtended by the red giant star as seen by the white dwarf star is approximately $0.13 \%-0.29 \%$ for a RG star between 27 and $40 R_{\odot}$ and a binary separation of 1.72 AU. However, the hydrodynamical studies of Mastrodemos \& Morris (1999) show that the density falls off steeply as a function of latitude, and is as low as $1 \%$ of the midplane density by latitudes of about $\pm 40^{\circ}$. 
This gives a scale height of about $9^{\circ}$. Thus, the red giant star subtends a much bigger fraction of the solid angle up to this scale height, i.e., to as much as $1.9 \%$.

Furthermore, studies of the supernova blast waves around red giant stars indicate that the blast wave diffracts around the RG star, with a hole in the debris of angular size $\sim 31^{\circ}-34^{\circ}$, which did not depend strongly on whether the companion star was indeed a RG star or a main-sequence or subgiant star (Marietta et al. 2000). In this case the fraction of the sky subtended by this hole in the debris field is $\sim 10 \%-13 \%$. If the material is concentrated in the midplane, the effect is substantially bigger as noted in the previous paragraph. Thus there are several reasons to expect that material in the shadow of the RG star can have an observable effect.

Stripping of material from the red giant companion by the blast wave for supernova has been studied by Wheeler et al. (1975) and by Livne et al. (1992); however, Lane et al. (2007) showed that stripping of material for the RG star is negligible for the less energetic blasts from RS Oph.

Another effect is the heating of the surface of the red giant star that faces the nova (see Appendices), as seen in Figure 8, for times past the maximum in the visible light curve. Our calculations indicate that this side of the red giant star increases from about $3400 \mathrm{~K}$ to as much as $4200 \mathrm{~K}$ within a few days past the outburst. The temperature steadily declines from day 70 until it reaches equilibrium with the other side around day 250 . The surface of the RG star is initially heated by the shock from the blast wave (not included in our calculations); however, the continued heating during the high-luminosity phase is important as it affects the interpretation of data from modern complex instrumentation such as stellar interferometers and adaptive optics systems, where various subsystems are controlled at wavelengths other than the measurement wavelength.

Figure 9 displays a schematic view of the system geometry from days 4-90 after the recent outburst. Assuming typical wind velocities of about $20 \mathrm{~km} \mathrm{~s}^{-1}$ for the red giant wind, there are roughly 17 rings separated by approximately 3.3 mas that form between RS Oph outbursts. The top left panel displays the system geometry at 4 days postoutburst. A gray ring is drawn in the center of the figure to indicate the size of the region affected by the blast at this epoch. In this panel the outer part of the spiral is overlaid with light gray to indicate that it is not known if the material stays in a coherent spiral past the first few turns. The diameter of the shocked region is about 8.8 mas assuming the blast wave travels at a velocity of $\leq 1800 \mathrm{~km} \mathrm{~s}^{-1}$ in the plane of the orbit, using the velocity measured by Chesneau et al. (2007) at approximately the same epoch as our measurements. (We acknowledge that most out-of-plane blast-wave velocities noted in the literature are much higher than this.) In this figure we assume the blast wave moves at constant velocity as it traverses the spiral shock material. We show the extreme case in which the blast wave is $100 \%$ efficient in sweeping up material, thus creating a ringlike structure that propagates outward from the system. Note this figure is meant only to be illustrative and differs in detail from estimates of the position of the blast wave from observations, such as O'Brien et al. (2006), who obtained a value of the shock radius of 8.6 mas at day 13.8 by which time the blast wave had apparently slowed considerably. We obtain a value of 10.5 mas for the shock radius assuming a constant velocity of $\sim 1800 \mathrm{~km} \mathrm{~s}^{-1}$ from one day past the initiation of the TNR process, which we take to be about 3 days before maximum light in $V$ band (Starrfield et al. 1985). The value of 8.6 mas at day 13.8 is consistent with a somewhat lower mean velocity, of the order of $1400 \mathrm{~km} \mathrm{~s}^{-1}$. It is beyond the scope of this paper to compute the evolution of the

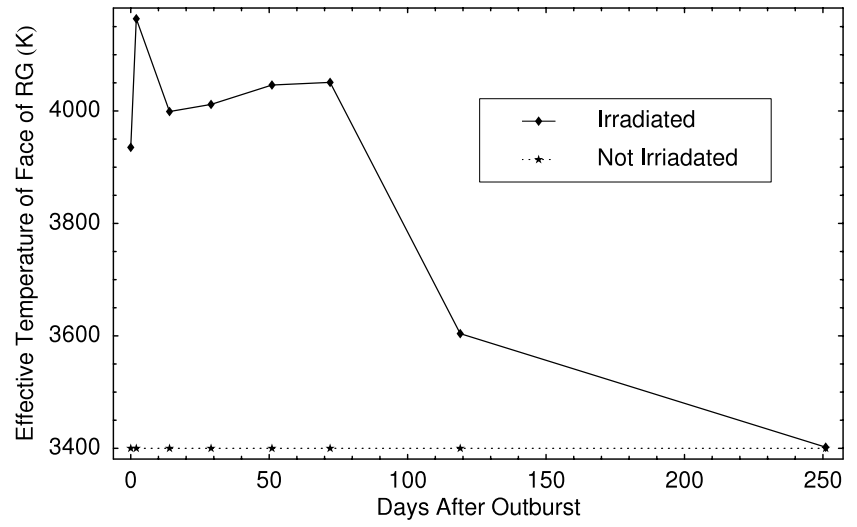

FIG. 8.- Temperature of red giant star for the surface that is facing the Nova as a function of the number of days postoutburst. Also plotted is the temperature of the nonirradiated side of the red giant. Modeled temperature rise is due to heating from irradiation by the nova and neglects any effect due to the passage of the forward shock.

blast wave; however, this figure makes a connection with the previous observational evidence for a "ring" of material concentrated in the plane of the orbit as discussed by Garcia (1986) and with the recent observations of Chesneau et al. (2007), who observe different velocities perpendicular to the plane of the orbit than in the plane of the orbit.

The evolution of the luminosity of the red giant and nova is also significant in aiding our understanding of the observations. Figure $10 a$ displays computed $V$-band light curves up to day 250 after the outburst including the effect of the irradiation of the accretion disk and the irradiation of the red giant star by the nova. Note this calculation overestimates the total luminosity of the nova during the period from about 10 days to about 50 days, and this is likely due to the simplified disk geometry that we have employed in our own calculations. Most importantly these light curves show that the $V$-band luminosity is dominated by the nova for about the first 50-70 days, and after that the red giant star dominates the $V$-band luminosity. This is significant as most telescopes track on $V$-band light (including interferometers) and the tracking center then moves by a mas or so during the postoutburst evolution of the system. $H$-band luminosity evolution is plotted in Figure $10 b$, and is different than that of the $V$-band evolution. At $H$ band the nova dominates the luminosity only for the first 5 days or so, and after that the $H$-band light curve is completely dominated by the red giant star. This means the phase center for fringe detection is offset from that of the tracking center from day 5 onward by a mas or so, as mentioned above. The $N$-band luminosity, displayed in Figure $10 c$, evolves like that of the $H$ band, and the nova dominates the mid-infrared light only up to day 4 . After that there is an offset between the tracking center and $N$-band fringe center like that noted for the $H$ band.

\section{IMPLICATIONS TO THE KECK NULLER AND OTHER HIGH ANGULAR RESOLUTION OBSERVATIONS}

We now reexamine data from the 2006 outburst within the framework of the spiral shock model of the geometry of RS Oph presented in the last section. In its simplest form, this geometry evolves into a bipolar morphology similar to that seen in the radio emission from the outburst that was discussed by O'Brien et al. (2006), as seen in the bottom right panel of Figure 9.

Such a geometry provides a natural explanation for some of the differences between the interferometric and other measurements, as the blast wave would be restricted and slowed in the orbital plane due to the high-density regions, while its flow would 


\section{RS Ophiuchi}

\section{Day 4}
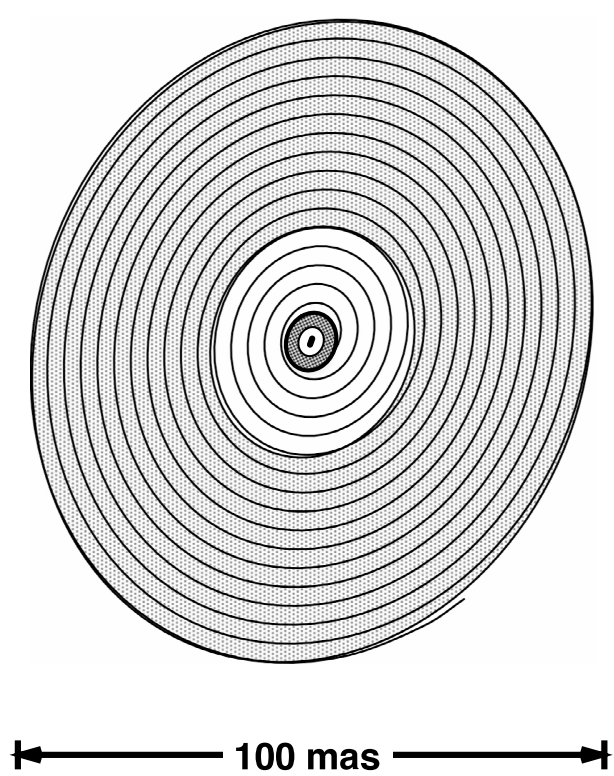

\section{RS Ophiuchi}

\section{Day 57}

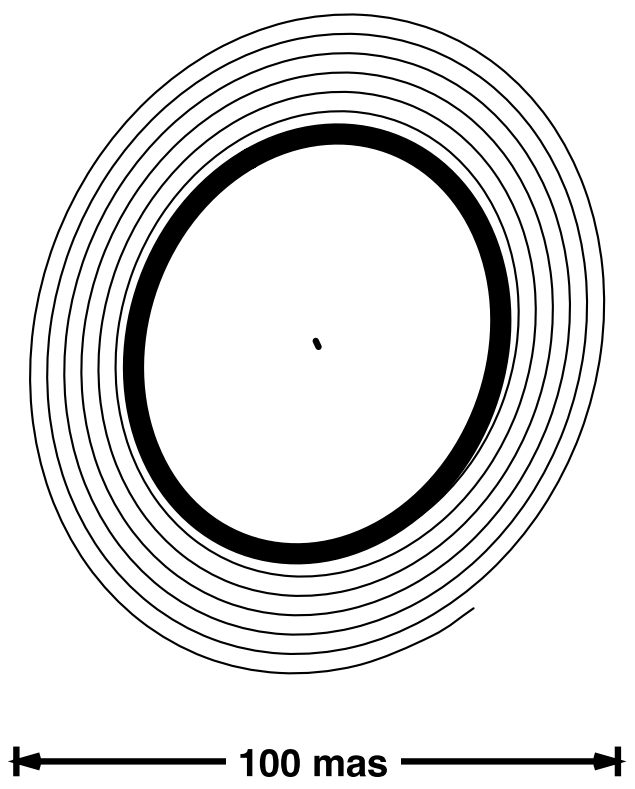

RS Ophiuchi

Day 21

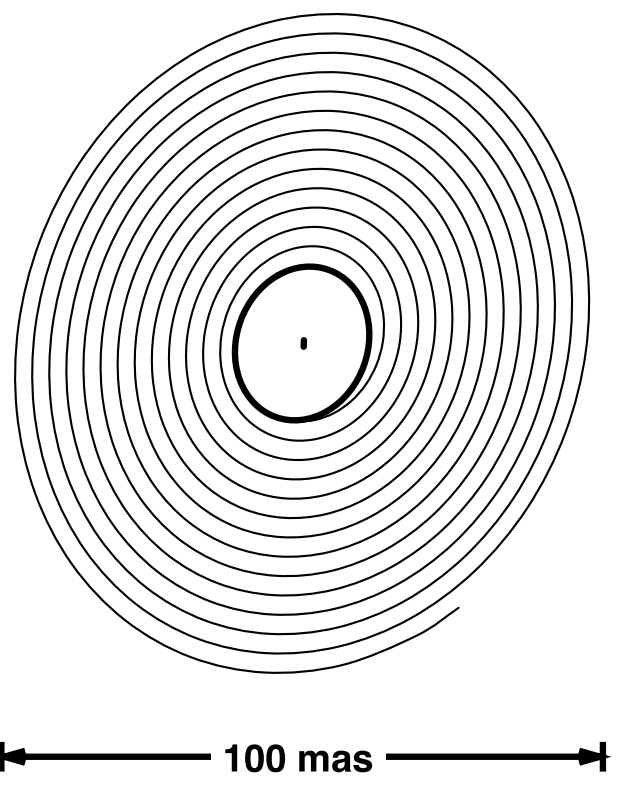

100 mas

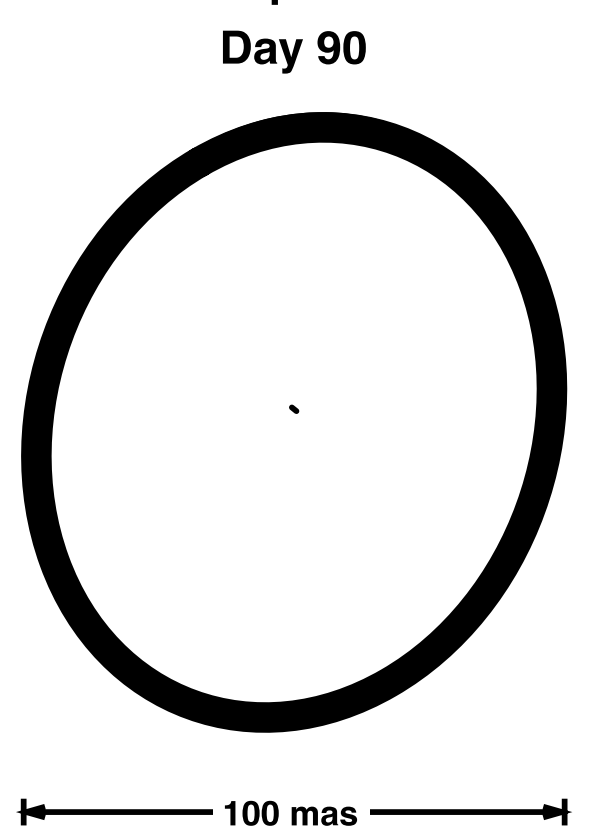

Fig. 9.- Spiral shock wave model of RS Oph. Top left: The system geometry at 4 days postoutburst. A gray ring is drawn in the center of the figure to indicate the size of the shocked region at this epoch. The outer part of the spiral is overlayed with light gray to indicate that it is not known if the material stays in a coherent spiral past the first several turns. The diameter of the shocked region is about 5 mas assuming the blast wave travels at a velocity of $1730 \mathrm{~km} \mathrm{~s}^{-1}$ in the plane of the orbit. Top right: The blast wave is now about 13 mas in diameter on day 21. Bottom left: The blast wave is about 36 mas in diameter on day 57 . Bottom right: By day 90 the blast wave has traversed the entire spiral pattern.

be relatively unimpeded perpendicular to that plane. This corresponds well to what was observed by Chesneau et al. (2007), where the observed velocity components came from distinctly different position angles. These authors also calculate that the red giant star should be at position angle $150^{\circ}-170^{\circ}$ at the time of the outburst. Recently, Brandi et al. (2008) have derived spectroscopic orbits for both components of the RS Oph system based on the radial velocities of the $\mathrm{M}$ giant absorption lines and the broad emission wings of $\mathrm{H} \alpha$. They have also constrained the orbit inclination, $i \geq 49 \pm 3$, using the estimated hot component 

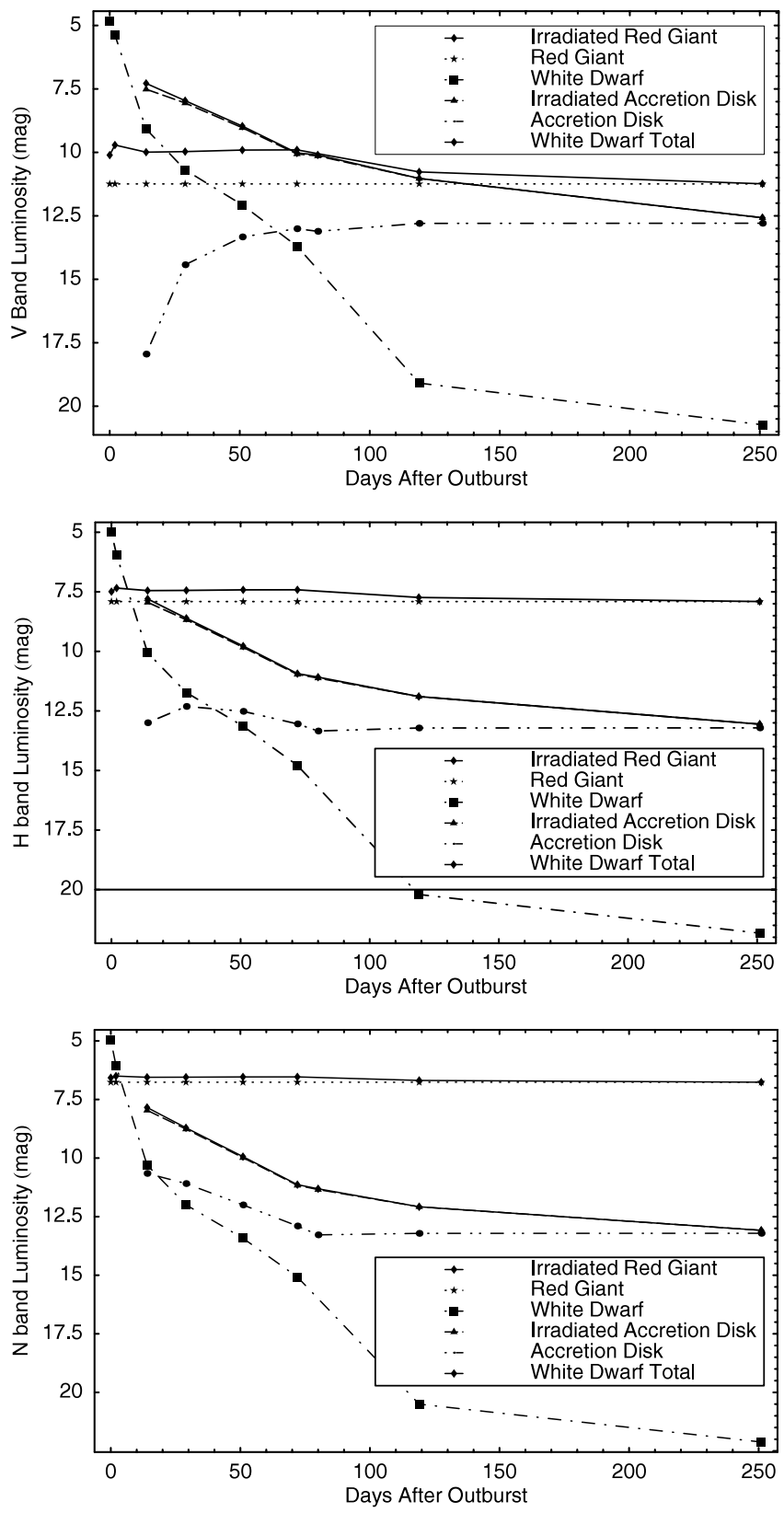

FIG. 10.-Top: $V$-band luminosity as a function of time for nova and red giant components of binary system. Middle: $H$-band luminosity evolution. Bottom: $\mathrm{N}$-band luminosity evolution.

mass, $M_{h} \sin ^{3} i=0.59 M_{\odot}$, and assuming that the white dwarf mass cannot exceed the Chandrasekhar limit. Thus the plane of the orbit is such that the high-velocity flow would be expected to be mostly east-west and the continuum emission would have an elliptical shape with the position angle measured by Chesneau et al. (2007). Furthermore, the spiral shock wave tends to have the largest densities near the white dwarf star and on the opposite side of the red giant star, the separation being of the order of $2-$ 4 times the separation between the two stars, i.e., a few mas. The larger separation occurs when the red giant star has been spun up so that it is synchronous with the orbital motion. Thus it is possible that some of the data can be interpreted in terms of emission from hot clumps of material within the spiral shocks. Detailed radiative transfer calculations will need to be performed to refine this model.
There is a wealth of recent observational work on RS Oph across the spectrum from X-ray (Sokoloski et al. 2006; Bode et al. 2006), near-infrared (Evans et al. 2007c; Chesneau et al. 2007; Das et al. 2006), radio (O'Brien et al. 2006), and now midinfrared (Evans et al. 2007a; Evans et al. 2007b; this work). Generally speaking, the observational picture is consistent with the shocked wind model as described by Bode \& Kahn (1985). Extensive modeling of the light curves has been performed by Hachisu \& Kato (2000); Hachisu \& Kato (2001) which include effects such as the irradiation of the red giant star by the white dwarf, and the accretion disk, which become important about 4 days postoutburst, and which are the basis for our own calculations presented in Figures 6, 7, and 10. In this general picture the recurrent nova differs from a classical nova in that the highvelocity ejecta from the WD is impeded by the wind from the red giant star, which in turn generates a shock wave that propagates through the red giant wind. Observations of the shock wave in the near-infrared by Das et al. confirm this picture and trace the evolution of the widths of $\mathrm{Pa} \beta$ and $\mathrm{O}_{\mathrm{I}}$ lines as a function of time postoutburst, and clearly show the expected free-expansion phase of the shock ended about 4 days postoutburst. This is also consistent with independent measurements conducted by Bode et al. (2006) in which this phase was proposed to have ended by approximately day 6 .

Most of the observational work has used spectroscopic methods, but only the interferometric observations are capable of spatially separating the various components of RS Oph that contribute to the emission that is seen spectroscopically. Chesneau et al. (2007) observed RS Oph 5.5 days postoutburst in the continuum at $2.13 \mu \mathrm{m}, \mathrm{Br} \gamma$ at $2.17 \mu \mathrm{m}$, and $\mathrm{He}$ I at $2.06 \mu \mathrm{m}$ with the AMBER instrument on the VLTI. They fitted their data with uniform ellipses, Gaussian ellipses, and a uniform ring. The models had excellent consistency in terms of the position angle of the ellipse $\left(\sim 40^{\circ}\right)$ and the ratio between minor and major axes $(0.6)$. For the continuum at $2.13 \mu \mathrm{m}$, a uniform ellipse had a major axis of $4.9 \pm 0.4$ and minor axis $3.0 \pm 0.3$ mas, while the Gaussian ellipse had a major axis of $3.1 \pm 0.2$ and minor axis of $1.9 \pm 0.3$ (FWHM). These measurements are consistent with the expected size of the shocked region at the epoch of their measurements.

Monnier et al. (2006) presented IOTA results in the nearinfrared bands at $H$ and $K$, and had somewhat different conclusions than some of the other workers because they were able to fit their visibility and closure phase data best with a binary model with two sources separated by $3.13 \pm 0.12$ mas, position angle of $36^{\circ} \pm 10^{\circ}$, and brightness ratio of $0.42 \pm 0.06$. Their Gaussian fits at $2.2 \mu \mathrm{m}$ gave a FWHM of $2.56 \pm 0.24$ mas for the same period of time as the AMBER observations. A striking feature of those results is that the size of the emitting region decreased $10 \%-20 \%$ between about days 4 and 65 postoutburst. For example, the size at $2.2 \mu \mathrm{m}$ actually decreased from about 2.6 to 2.0 mas (FWHM), while at $1.65 \mu \mathrm{m}$, the size decreased from 3.3 to 2.9 mas. However, the $2 \mu \mathrm{m}$ continuum sizes are in approximate agreement. Monnier et al. (2006) rule out an expanding fireball model, however, they would have overresolved the fireball anyway since it would be about 8.8 mas in diameter, at a distance of $1600 \mathrm{pc}$, or a substantially larger angular size if the distance were smaller. Lane et al. (2007) observed RS Oph also with IOTA, PTI, and the Keck Interferometer at $H$ band over a longer time period, up to 120 days from the $V$-band maximum. They observed an increase in diameter from approximately 3-4 mas from days $0-20$ and a decrease to less than 2 mas in diameter around day 120. They interpreted the near-infrared size data in terms of a simplified model of free-free emission in the postnova 
wind as the mass ejected in the wind decreased during that time period.

The emitting region at $10 \mu \mathrm{m}$ is somewhat larger than that seen at $2 \mu \mathrm{m}$; for example, our data are fitted by a Gaussian $4.0 \pm 0.4$ mas (FWHM), while the IOTA data had a size of $2.6 \pm 0.2$ mas. By 4 days postoutburst, a spherical shock wave would be expected to have a radius of about 3.9 mas assuming a speed of approximately $1730 \mathrm{~km} \mathrm{~s}^{-1}$ (O'Brien et al. 2006) and a distance of $1.4 \mathrm{kpc}$. Thus, according to this simple model, all the interferometric IR continuum emission should be coming from within the postshock region. The AMBER/VLTI results indicate a more complex picture of the velocity field of the expanding material, with two indicated-a "slow" field between -1800 and $1800 \mathrm{~km} \mathrm{~s}^{-1}$, and a "fast" one between -3000 and $-1800 \mathrm{~km} \mathrm{~s}^{-1}$ and 1800 and $3000 \mathrm{~km} \mathrm{~s}^{-1}$. The position angle of the emitting material for the two velocity groups differ, with the "fast" component being well defined in the east-west (position angle $90^{\circ}$ or $270^{\circ}, \pm 5^{\circ}$ ) direction and the "slow" component with position angles from $55^{\circ}-110^{\circ}$ to modulo $180^{\circ}$.

Recent observations by Bode et al. (2007) using the HST confirm the elliptical shape seen with AMBER, with an axial ratio of 0.5-0.6. The HST observations also confirm the velocity structure seen by AMBER, i.e., the "fast" velocity field in the eastwest direction, of the order of $3000 \mathrm{~km} \mathrm{~s}^{-1}$, and the "slow" velocity about half that of the "fast" one. The HST results showed that the expansion rate in the plane of orbit of the stars decreased, from about 0.62 mas day $^{-1}$ on day 13.8 to 0.48 mas day $^{-1}$ on day 155. This is a reduction in velocity from about 1700 to about $1300 \mathrm{~km} \mathrm{~s}^{-1}$.

The spiral shock model is clearly relevant to the interpretation of the IOTA data presented in Monnier et al. (2006). As noted above, the increased density of gas and dust in the arms of the spiral would certainly provide an impediment to the free expansion of the fireball, as well as provide a reservoir of hot material that would emit strongly at $2 \mu \mathrm{m}$. Their data indicate a closure phase that is consistent with zero or $180^{\circ}$ for the first epoch (days 4-11) and convincingly nonzero only for hour angles from -1.5 to $-1 \mathrm{hr}$ on the second epoch (days 14-29), and at $+\frac{1}{2} \mathrm{hr}$ on the third epoch (days 49-65). Hence, the closure phase signals and the binary interpretation may be more consistent with hot clumpy material that is cooling, some of which may (re)condense into dust as the white dwarf's outburst luminosity declines from its most luminous state, i.e., as shown in Figure 6. Note that the sublimation radius decreases from $\sim 5$ mas to about 2 mas from day 70 to day 120. Furthermore, as the luminosity changes, the star tracker on IOTA may be providing a different optical center to the fringe detection system, since in the first few days during the outburst the optical emission is dominated by that centered on the white dwarf star itself, whereas after it has cooled, the optical emission is dominated by the red giant star. Thus the effects of changes in the optical tracking and interferometric phase center will need to be included in the analysis to properly interpret the data. The offset between the optical tracking center and the fringe phase center can cause a miscalibration of the visibility. Since the $2 \mu \mathrm{m}$ emitting region is actually decreasing in size with time, as seen in their Figure 1 and Table 2, it seems more likely they are observing the cooling of this hot material near the two stars than actually resolving the binary, however, the effects of the material in the shadow of the red giant star must be included in the interpretation of the near-infrared and mid-infrared data.

\section{SUMMARY AND CONCLUSIONS}

We analyzed data from the recurrent nova RS Oph for the epoch at $\sim 4$ days postoutburst using the new KIN instrument.
These data allowed us to determine the size of the emitting region around the RS Oph at wavelengths from 8-12 $\mu \mathrm{m}$. By fitting the unique KIN inner and outer spatial regime data, we obtained an angular size of the mid-infrared continuum of $6.2,4.0$, or 5.4 mas for a disk profile, Gaussian profile (FWHM), and shell profile, respectively. The data show evidence of enhanced neutral atomic hydrogen emission located in the inner spatial regime relative to the outer regime. There is also evidence of a $9.7 \mu \mathrm{m}$ silicate feature seen outside of this region, which is consistent with dust that had condensed prior to the outburst, and which has not yet been disturbed by the blast wave from the nova. Our analysis of the observations, including the new ones presented in this paper, are most consistent with a new physical model of RS Oph, in which spiral shock waves associated with the motion of the two stars through the cool wind from the red giant create density enhancements within the plane of their orbital motion.

Further observations are needed to clarify this new picture of the RS Oph system. One issue that has not been fully resolved is whether or not the red giant star really overflows its Roche lobe. If so a hot spot would be expected where the material streaming from the red giant envelope hits the accretion disk, and UV or $\mathrm{X}$-ray observations could search for this effect. Another approach would be to observe RS Oph over several orbital cycles using infrared photometry to look for variations of the light curve due to the departure of the red giant star from spherical symmetry. High-resolution spectra could also help. Confirmation of the rotational velocity of the red giant star measured by Zamanov et al. (2007) would be worthwhile, and could provide another estimate of the absolute size of the red giant star, assuming that it is corotating with the orbit. Further KIN observations within the next few years would also be helpful as they could show evidence of the reestablishment of the spiral shock wave, and perhaps some information about the shape of the circumstellar material and dust formation. Another epoch of HST observations would also determine the deceleration of the outflow in the two directions, i.e., within the plane of the orbit of the two stars and along the poles.

Theoretical studies of the motion of the blast wave in an environment with a high-density region in the plane of the orbit of the two stars are also worthwhile. In particular, it would be important to understand how the blast wave is diffracted around the red giant star and how it propagates in an medium with the periodic density enhancements in the plane due to the spiral pattern.

The recurrent nova RS Ophiuchi is a rich system for the study of circumstellar matter under extreme physical conditions. Continued study will provide important insights into Type Ia supernovae, of which RS Oph may be a progenitor.

We are grateful to the National Aeronautics and Space Administration, Jet Propulsion Laboratory, the California Association for Research in Astronomy, the Harvard-Smithsonian Center for Astrophysics (including SAO grant G06-7022A to J. L. S.), and to the National Aeronautics and Space Administration, Goddard Space Flight Center for support of this research. The data presented herein were obtained at the W. M. Keck Observatory, which is operated as a scientific partnership among the California Institute of Technology, the University of California and the National Aeronautics and Space Administration. The Observatory was made possible by the generous financial support of the W. M. Keck Foundation. J. P. W. acknowledges support provided by an NPP Fellowship (NNH06CC03B) at 
NASA Goddard Space Flight Center. This work has made use of services produced by the Michelson Science Center at the California Institute of Technology. One of the authors (R. K. B.) would also like to acknowledge Eugene E. Rudd formerly of the United States Naval Research Laboratory for his continued support and encouragement. The authors thank the referee, Michael Bode, for his careful and thorough review of the manuscript, which has helped us to significantly improve it.

Facilities: Spitzer, IOTA, Keck:I, Keck:II

\section{APPENDIX}

\section{LUMINOSITY EVOLUTION}

In this appendix we discuss the mathematical formulation used to derive the evolution of the luminosity of the white dwarf star starting at the maximum magnitude in $V$ band. Our calculations are based on those presented in the paper by Hachisu \& Kato (2001), who expanded on their discussion of their light-curve model presented in Hachisu \& Kato (2000).

Our purpose is to evaluate the light curve not only at $V$ band, but also at $H, K$, and $N$ bands, which have been used by the AMBER, IOTA, Keck, and PTI interferometers to observe RS Oph after the 2006 outburst. Our discussion is in fact relevant to any modern instrument that has wave front sensing and control, and also tracking, at wavelengths different than that of the observations of interest. For example, the IOTA interferometer performs its precision pointing at $V$ band, but observations are made at $H$ band. Similarly the Keck Interferometer does precision pointing at $V$ band, wave front control of the two large apertures at $H$ band, and the data are taken at $N$ band. This difference in wavelengths is important because as the $V$-band luminosity of the white dwarf star decreases, the observed light from the binary is mainly from the red giant star, which gives a shift in the center of light. This shift can affect the calibration of these instruments as there is an offset, which will change over time as compared to a calibrator, which has all the same optical center at all times for all of these wave bands.

The evolution of the stellar parameters for the white dwarf are given in Table 5 of Hachisu \& Kato (2001). We assume the parameters for the red giant star remain constant with $R_{\mathrm{RG}}=40 R_{\odot}$, and $T_{\mathrm{RG}}=3400 \mathrm{~K}$.

The blackbody flux density (ergs $\mathrm{cm}^{-2} \mathrm{~s}^{-1} \mathrm{~Hz}^{-1}$ ) at a frequency, $\nu$, for a star of temperature, $T_{*}$, radius, $R_{*}$, and distance, $D$ is

$$
F_{*}(\nu)=2 \pi\left(R_{*} / D\right)^{2}\left(h \nu^{3} / c^{2}\right) /\left[\exp \left(h \nu / k T_{*}\right)-1\right],
$$

where $h, k$, and $c$, are Planck's constant, the Boltzmann constant, and the speed of light, respectively.

The total luminosity $\left(\mathrm{ergs} \mathrm{s}^{-1}\right)$ is

$$
L_{*}=4 \pi \sigma R_{*}^{2} T_{*}^{4},
$$

where $\sigma$ is Stefan's constant.

\section{A1. SUBLIMATION RADIUS}

The temperature of a black grain in radiative equilibrium at a distance, $r$, from a star with luminosity, $L_{*}$ is

$$
T_{\text {grain }}=\left(\frac{L_{*}}{16 \pi \sigma r^{2}}\right)^{1 / 4}=\frac{T_{*}}{\sqrt{2}}\left(\frac{R_{*}}{r}\right)^{1 / 2} \text {. }
$$

A simple rearrangement of the above equation yields a formula for the sublimation radius, $R_{\text {sub }}$, assuming a sublimation temperature, $T_{\text {sub: }}$ :

$$
R_{\text {sub }}=\left(\frac{L_{*}}{16 \pi \sigma T_{\text {sub }}^{4}}\right)^{1 / 2}=\frac{R_{*}}{2}\left(\frac{T_{*}}{T_{\text {sub }}}\right)^{2} .
$$

For this paper we assume a sublimation temperature of $1500 \mathrm{~K}$.

The evolution of the sublimation radius for the red giant star (dashed lines) and nova (solid lines) beginning at the maximum in $V$ band is displayed in Figure 7. Note that the sublimation radius remains approximately constant at about 5 mas for the first 70 days, after which it gradually reduces to $<0.2$ mas after about day 120 .

\section{A2. ILLUMINATION OF THE RED GIANT STAR}

During the high-luminosity phase of the outburst, the surface of the red giant star facing the nova is heated substantially. We calculate the effect of the nova luminosity on the red giant star using equation (10) of Hachisu \& Kato (2001), which is based on thermal equilibrium between the faces of the two stars, which we display here:

$$
\sigma T_{\mathrm{RGI}}^{4}=\eta_{\mathrm{RG}} \cos (\theta) L_{\mathrm{WD}} /\left(4 \pi r^{2}\right)+\sigma T_{\mathrm{RG}}^{4}
$$

where $T_{\mathrm{RGI}}$ is the new effective temperature of the red giant star for facing side and where we include an extra term from the white dwarf luminosity, $L_{\mathrm{WD}}$. There are two additional constants included in this new term, the first is $\eta_{\mathrm{RG}}$, which is an effective emissivity for the 
stellar surface, while the second, $\cos (\theta)$ is the average inclination angle of the surface. The term $r$ is the distance between the two stars, and $T_{\mathrm{RG}}$ is the original temperature of the red giant star. Equation (A5) can be rearranged into a simple form after substituting for $L_{\mathrm{WD}}$ :

$$
T_{\mathrm{RGI}}^{4}=\left[\eta_{\mathrm{RG}} \cos (\theta)\left(\frac{R_{\mathrm{WD}}}{r}\right)^{2}\right] T_{\mathrm{WD}}^{4}+T_{\mathrm{RG}}^{4} .
$$

Equation (A5) was evaluated as a function of time, using the evolution of the white dwarf luminosity derived from equation (A2) and the values from Table 5. The result of our computation of this effect is displayed in Figure 8, in which we use $r=325 R_{\odot}$, $\cos (\theta)=0.5, \eta_{\mathrm{RG}}=0.5$, and $T_{\mathrm{RG}}=3400 \mathrm{~K}$.

\section{A3. ACCRETION LUMINOSITY}

The computation of the evolution of the accretion luminosity is based on the treatment of Hachisu \& Kato (2001) with the simplification of a flat accretion disk, instead of adding the complexity of a warped accretion disk that is used in their paper. These calculations are based on the well-known Lynden-Bell \& Pringle (1974) and Pringle (1981) equations for the luminosity of an accretion disk, where the accretion luminosity is based on a numerical integration of the flux from the accretion disk, assuming a temperature distribution across the disk. Normally the temperature of the accretion disk is determined solely by the accretion rate onto the disk for normal viscous heating of the disk. To this Hachisu \& Kato (2001) added a term based on additional heating of the disk from the white dwarf star as it is in a high-luminosity state and is contracting and heating during the constant high-luminosity period after the outburst. The flux density of the disk at frequency $\nu$ is given by

$$
F_{\text {disk }}(\nu)=\frac{2 \pi \cos \left(\theta_{\text {inc }}\right)}{D^{2}} \int_{R_{\text {in }}}^{R_{\text {out }}} \rho B_{\nu}\left[T_{\text {disk }}(\rho)\right] d \rho
$$

The conventional expression for the radial distribution of the temperature of the disk is

$$
T_{\text {disk } 1}(\rho)=\left\{\frac{3 G M_{*} \dot{M}}{8 \pi \sigma \rho^{3}}\left[1-\left(\frac{R_{*}}{\rho}\right)^{1 / 2}\right]\right\}^{1 / 4},
$$

where $T_{\text {disk } 1}$ is the temperature in the accretion disk as a function of radius $\rho$ in the plane of the disk, $\theta_{\text {inc }}$ is the inclination angle of the disk, and $D$ is the distance to the Earth (Pringle 1981). The quantity $B_{\nu}$ is the Planck function, $M_{*}$ is the mass of the white dwarf, $R_{*}$ is its radius, $\dot{M}$ is the accretion rate onto the disk, and $\sigma$ and $G$ are the Stefan-Boltzmann constant and the gravitational constant, respectively. The maximum temperature in the accretion disk occurs at a radius $R_{\max }=1.36 R_{*}$, and is given by

$$
T_{\max }=0.488\left(\frac{3 G M_{*} \dot{M}}{8 \pi \sigma R_{*}^{3}}\right)^{1 / 4},
$$

as discussed in Lynden-Bell \& Pringle (1974), Pringle (1981), and Hartmann (1998). In this treatment we neglect the emission from the boundary layer, which occurs over a very small angular region around the surface of the white dwarf star and emits at a very high temperature, which would have a negligible contribution to the luminosity in the infrared region near $10 \mu \mathrm{m}$. The evolution of the luminosity of the accretion disk will be computed using the formulation of Hachisu \& Kato (2001), as set up below.

Let us now formulate the evolution of the accretion disk luminosity by adjusting some of the parameters in the equation above as a function of time. In particular the outer radius of the accretion disk $R_{\text {disk }}$ is parameterized by a power law decrease from day 7 until day 79 using the following formulae:

$$
\begin{gathered}
R_{\mathrm{disk}}=\alpha R_{1}^{*}, \\
\alpha=\alpha_{0}\left(\frac{\alpha_{1}}{\alpha_{0}}\right)^{\left(t-t_{0}\right) / 72} .
\end{gathered}
$$

In these equations $R_{1}^{*}$ is the inner critical radius of the Roche lobe for the white dwarf (nova) component, which we assume is $138.6 R_{\odot}$

The parameter $\alpha$ helps define the size of the accretion disk which varies between $\alpha_{0}=0.1$ and $\alpha_{1}=0.008$, and so the power-law form of equation (A10) is to make an interpolation function. Haschisu \& Kato include irradiation of the accretion disk as the WD luminosity changes between days 7 and 79 , where the accretion disk temperature is now given by

$$
\sigma\left[T_{\text {disk }}(\rho)\right]^{4}=\eta_{\text {disk }} \cos \left(\theta_{\text {inc }}\right)\left(\frac{L_{\mathrm{WD}}}{4 \pi \rho^{2}}\right)+\left\{\frac{3 G M_{*} \dot{M}}{8 \pi \rho^{3}}\left[1-\left(\frac{R_{*}}{\rho}\right)^{1 / 2}\right]\right\} .
$$

The second term of this equation is the traditional accretion luminosity term from equation (8), while the first term includes a contribution from the luminosity of the white dwarf. The parameter $\eta_{\text {disk }}$ is an efficiency factor, and is assumed to be 0.5 , and $\cos \left(\theta_{\text {inc }}\right)$ is 0.1 , based on the average inclination of the surface. They assume the outer radius of the accretion disk is at a temperature of $2000 \mathrm{~K}$, 
and is not affected by radiation from the WD photosphere. For the remainder of the calculation we will compute $\alpha$ in discrete time intervals corresponding to what we have done before over the days that this effect matters.

The integral is numerically evaluated at the center wavelengths for $V(0.55 \mu \mathrm{m}), H(1.65 \mu \mathrm{m})$, and $N(10.5 \mu \mathrm{m})$ bands, using an inner radius given by the radius of the white dwarf star and the outer radius given by the equation for $R_{\text {disk}}$, for the time steps of Table 5 and the white dwarf parameters in that table. The results are plotted in Figure 10. In this figure the quantities plotted include the contributions of the red giant and white dwarf alone (stars and solid squares), the red giant including effects of irradiation by the white dwarf (diamonds), the accretion disk alone (solid circles and dash-double-dotted line), the irradiated accretion disk (triangles and dashed line), and the total for the white dwarf including the irradiated accretion disk (diamonds and solid line).

Adams, W. S., \& Joy, A. H. 1933, PASP, 45, 249

Barbon, R., Mammano, A., \& Rosino, L. 1969, Comm. Konkoly Obs., 65, 257

Barry, R. K., Mukai, K., Sokoloski, J. L., Danchi, W. C., Hachisu, I., Evans, A., Gehrz, R., \& Mikolajewska, J. 2008a, in ASP Conf. Ser., RS Ophiuchi (2006) and the Recurrent Nova Phenomenon, ed. A. Evans, M. F. Bode, \& T. J. O'Brien (San Francisco: ASP), in press

Barry, R. K., Skopal, A., \& Danchi, W. C. 2008b, ApJ, in press

Bode, M. F., Harman, D. J., O’Brien, T. J., Bond, H. E., Starrfield, S., Darnley, M. J., Evans, A., \& Eyres, S. P. S. 2007, ApJ, 665, L63

Bode, M. F., \& Kahn, F. D. 1985, MNRAS, 217, 205

Bode, M. F., et al. 2006, ApJ, 652, 629

Brandi, E., Quiroga, C., Ferrer, O. E., Mikołajewska, J., \& Garcia, L. G. 2008, in ASP Conf. Ser., RS Ophiuchi (2006) and the Recurrent Nova Phenomenon, ed. A. Evans, M. F. Bode, \& T. J. O'Brien (San Francisco: ASP), in press Chesneau et al. 2007, A\&A, 464, 119

Colavita, M. M. Serabyn, G. Wizinowich, P. L., \& Akeson, R. L. 2006, Proc. SPIE 6268, 3

Das, R., Banerjee, D. P. K., \& Ashok, N. M. 2006, ApJ, 653, L141

Dobrzycka, D., \& Kenyon, S. J. 1994, AJ, 108, 2259

Evans, A., et al. 2007a, ApJ, 671, L157

2007b, MNRAS, 374, 1 2007c, ApJ, 663, L29

Eyres, S. P. S., et al. 1998, Ap\&SS, 255, 361

Fekel, F. C., Joyce, R. R., Hinkle, K. H., \& Skrutskie, M. F. 2000, AJ, 119 , 1375

Garcia, M. R. 1986, AJ, 91, 1400

Grevesse, N. 1984, Phys. Scr., 8, 49

Fleming, W. 1904, Harvard Coll. Obs. Circ., 76

Hachisu, I., \& Kato, M. 2000, ApJ, 536, L93

$$
\text { 2001, ApJ, 558, } 323
$$

Hartmann, L. 1998, Accretion Processes in Star Formation, (Cambridge: Cambridge Univ. Press)

Kenyon, S. J., \& Fernandez-Castro, T. 1987, AJ, 93, 938

Koresko, C., Colavita, M., Serabyn, E., Booth, A., \& Garcia, J. 2006, Proc. SPIE, 6268, 39

Lane, B. F., et al. 2007, ApJ, 658, 520

Livne, E., Tuchman, Y., and Wheeler, J.C., 1992, ApJ, 399, 665

Lynch, D. K., et al. 2006, ApJ, 638, 987

Lynden-Bell, D., \& Pringle, 1974, MNRAS, 168, 603
REFERENCES

Marietta, E., Burrows, A., \& Fryxell, B., 2000, ApJS, 128, 615

Mastrodemos, N., \& Morris, M. 1999, ApJ, 523, 357

Mauron, N., \& Huggins, P. J. 2006, A\&A, 452, 257

Monnier, J. D., et al. 2006, ApJ, 647, L127

Morris, M., Sahai, R., Matthews, K., Cheng, J., Lu, J., Claussen, M., \& SanchezContreras, C. 2006, in IAU Symp. 234, Planetary Nebulae in our Galaxy and Beyond, ed. M. J. Barlow \& R. H. Mendez (Cambridge: Cambridge Univ. Press), 469

Morrison, W. 1985, IAU Circ., 4030, http://www.cfa.harvard.edu/iauc/04000/04030 .html\#Item0

Mürset, U., \& Schmid, H. M. 1999, A\&AS, 137, 473

Narumi, H., Hirosawa, K., Kanai, K., Renz, W., Pereira, A., Nakano, S., Nakamura, Y., \& Pojmanski, G. 2006, IAU Circ., 8671, 2

O’Brien, T. J., et al. 2006, Nature, 442, 279

Oppenheimer, B. D., \& Mattei, J. A. 1993, J. AAVSO, 22, 1050

Payne-Gaposchkin, C. 1957, The Galactic Novae (Amsterdam: North-Holland) Pottasch, S. R. 1967, Bull. Astron. Inst. Netherlands, 19, 227

Pringle, J. E. 1981, ARA\&A, 19, 137

Sacuto, s., Chesneau, O., Vannier, M., \& Cruzalebes, P. 2007, A\&A, 465, 469

Schaefer, B. 2004, IAU Circ. 8396, http://www.cfa.harvard.edu/iauc/08300/08396 .html\#Item2

Schild, H., Eyres, S. P. S., Salama, A., \& Evans, A. 2001, A\&AS, 378, 146

Serabyn, E., Colavita, M. M., \& Beichman, C. A. 2000, in ASP Conf. Ser. 196, Thermal Emission Spectroscopy and Analysis of Dust, Disks, and Regoliths, ed. M. L. Sitko, A. L. Sprague \& D. K. Lynch (San Francisco: ASP), 357

Serabyn, E., et al. 2004, Proc. SPIE, 5491, 806

- 2005, in Proc. SPIE, 5905, 272

- 2006, Proc. SPIE 6268, 37

. 2008, ApJ, in press

Sokoloski, J. L., Luna, G. J. M., Mukai, K., \& Kenyon, S. J. 2006, Nature, 442, 276

Starrfield, S., Sparks, W. M., \& Truran, J. W. 1985, ApJ, 291, 136

Wallerstein, G. 1958, PASP, 70, 537

Warner, B. 1995, Cataclysmic Variable Stars (Cambridge: Cambridge Univ. Press)

Wheeler, J. C., Lecar, M., \& McKee, C. F. 1975, ApJ, 200, 145

Zamanov, R. K., Bode, M. F., Melo, C. H. F., Bachev, R., Gomboc, A., Stateva, I. K., Porter, M., \& Pritchard, J. 2007, MNRAS, 380, 1053 